\title{
Sculpting humoral immunity through dengue vaccination to enhance protective immunity
}

\section{Wayne D. Crill*, Holly R. Hughes ${ }^{\dagger}$, Nicole B. Trainor, Brent S. Davis, Matt T. Whitney and Gwong-Jen J. Chang*}

Arboviral Diseases Branch, Division of Vector-Borne Diseases, Centers for Disease Control and Prevention, Public Health Service, U.S. Department of Health and Human Service, Fort Collins, CO, USA

\section{Edited by:}

Ken J. Ishii, National Institute of Biomedical Innovation, Japan

\section{Reviewed by:}

Alice Nyakeriga, Texas Tech University, USA

Qibin Leng, Institut Pasteur of Shanghai, Chinese Academy of Sciences, China

\section{*Correspondence:}

Wayne D. Crill and Gwong-Jen J. Chang, Arboviral Diseases Branch, Division of Vector-Borne Diseases, Centers for Disease Control and Prevention, Public Health Service, U.S. Department of Health and Human Service, P. O. Box 2087, 3156 Rampart Rd., Fort Collins, CO 80521 , USA.

e-mail:wcrill@cdc.gov; gxc7@cdc.gov ${ }^{\dagger}$ Present address:

Holly R. Hughes, Florida Department of Health, Tampa, FL, USA.
Dengue viruses (DENV) are the most important mosquito transmitted viral pathogens infecting humans. DENV infection produces a spectrum of disease, most commonly causing a self-limiting flu-like illness known as dengue fever; yet with increased frequency, manifesting as life-threatening dengue hemorrhagic fever (DHF). Waning cross-protective immunity from any of the four dengue serotypes may enhance subsequent infection with another heterologous serotype to increase the probability of DHF. Decades of effort to develop dengue vaccines are reaching the finishing line with multiple candidates in clinical trials. Nevertheless, concerns remain that imbalanced immunity, due to the prolonged prime-boost schedules currently used in clinical trials, could leave some vaccinees temporarily unprotected or with increased susceptibility to enhanced disease. Here we develop a DENV serotype 1 (DENV-1) DNA vaccine with the immunodominant cross-reactive B cell epitopes associated with immune enhancement removed. We compare wild-type (WT) with this cross-reactivity reduced (CRR) vaccine and demonstrate that both vaccines are equally protective against lethal homologous DENV-1 challenge. Under conditions mimicking natural exposure prior to acquiring protective immunity, WT vaccinated mice enhanced a normally sub-lethal heterologous DENV-2 infection resulting in DHF-like disease and $95 \%$ mortality in AG129 mice. However, CRR vaccinated mice exhibited redirected serotypespecific and protective immunity, and significantly reduced morbidity and mortality not differing from naïve mice. Thus, we demonstrate in an in vivo DENV disease model, that non-protective vaccine-induced immunity can prime vaccinees for enhanced DHFlike disease and that CRR DNA immunization significantly reduces this potential vaccine safety concern. The sculpting of immune memory by the modified vaccine and resulting redirection of humoral immunity provide insight into DENV vaccine-induced immune responses.

Keywords: dengue, dengue vaccines, DNA vaccines, immune refocusing, vaccine safety, antibody-dependent enhancement, original antigenic sin, dengue hemorrhagic fever

\section{INTRODUCTION}

Dengue viruses (DENV) are the most prevalent arthropod-borne viral pathogens infecting humans. These mosquito-transmitted viruses, members of the Flaviviridae, are endemic in most tropical and sub-tropical countries with nearly half of the world's population living at risk of DENV infection and resulting in over a million estimated infections annually (Gubler, 2002; Mackenzie et al., 2004). Infection with DENV can cause a broad range of symptoms, ranging from subclinical, to the self-limiting flu-like illness dengue fever, to the more severe and life-threatening dengue hemorrhagic fever and shock syndrome (DHF/DSS), characterized by increased vascular permeability producing plasma leakage, severe thrombocytopenia and hypotension leading to circulatory collapse (Gubler, 2006). DENV prevalence, infection rates, and disease severity have increased exponentially since the middle of the last century (Guzman et al., 2010). Despite decades of interest, need, and effort there remains no available dengue vaccine (Guy et al., 2010; Miller, 2010; Murphy and Whitehead, 2011; Thomas, 2011).
Dengue vaccine development faces both biological and immunological challenges. These include the necessity for a multivalent vaccine inducing balanced immunity, the lack of an animal model for DENV disease, and concerns regarding vaccine-induced immunopathology (Murphy and Whitehead, 2011; Heinz and Stiasny, 2012). DENV immune responses are both protective and pathogenic and this duality challenges vaccine development (Rothman, 2004). There are four closely related yet phylogenetically distinct DENV serotypes (DENV-1, -2, -3, and -4) and infection with any one serotype appears to induce lifelong serotype-specific immunity yet cross-protection between serotypes is limited and transient (Sabin, 1952; Kuno, 2003). Thus, in endemic regions individuals may be susceptible to up to four different DENV infections. Although there are a number of risk factors associated with DHF such as virus and host genetics, the strongest risk factor for severe dengue pathology is secondary infection with a previously unencountered (heterologous) serotype (Murphy and Whitehead, 2011). This association 
explains the exponential increase of DHF/DSS in recent decades as co-circulation and simultaneous transmission of the four DENV serotypes increases both temporally and geographically (Mackenzie et al., 2004; Gubler, 2006; Guzman et al., 2010). In humans, increasing viral load correlates with DENV disease severity and a large body of evidence points to the importance of immune enhancement being a causal factor for the increased viral loads associated with DHF (Vaughn et al., 2000; Libraty et al., 2002).

Two distinct yet not mutually exclusive mechanisms of immune enhancement explain the pathogenic manifestations characterizing DHF/DSS. The leading hypothesis is that DHF occurs via antibody-dependent enhancement (ADE) of infection, a phenomenon originally described for flaviviruses but later found to occur with diverse pathogens (Hawkes, 1964; Halstead, 1989; Morens, 1994; Thomas et al., 2006). Preexisting cross-reactive antibodies from a previous infection (or maternal antibodies in infants) recognize and bind to heterologous virus in a secondary infection (primary infection in infants), yet are unable to neutralize this virus, either because they are non-neutralizing, or for a lack of sufficient avidity or occupancy (Pierson et al., 2008). However, these non-neutralizing antibody-virus complexes increase the infection of monocytes via their Fc receptors, dramatically increasing viral replication and load, thereby causing DHF. Weakly- and non-neutralizing cross-reactive antibodies induced from immunodominant B cell epitopes are known to comprise the majority of the humoral immune response to DENV infection (Lai et al., 2008; Crill etal., 2009). In a recently developed type I/type II IFN receptor knock-out mouse model (AG129) of DENV disease (Williams et al., 2009), sub-neutralizing levels of homologous or heterologous DENV immune mouse sera (Balsitis et al., 2010; Zellweger et al., 2010) or cross-reactive human monoclonal antibodies (MAbs; Beltramello et al., 2010) enhanced viral replication in vivo producing a DHF-like disease.

The second, related mechanism of immune enhanced DENV pathology, also a generalized phenomenon, posits that there is a highly skewed cellular response to heterologous DENV infection driven by low affinity, cross-reactive memory CD4+ and CD8+ T cells (Brehm et al., 2002; Welsh and Selin, 2002; Welsh et al., 2004). Cross-reactive $\mathrm{T}$ cell epitopes have been identified across the DENV proteome, however, immunodominant CD8+ $\mathrm{T}$ cell epitopes in non-structural protein NS3 appear to be those most strongly associated with DHF (Mathew and Rothman, 2008; Duangchinda et al., 2010; Friberg et al., 2011).

The common theme throughout DENV immune enhancement is the concept of "original antigenic sin" describing a phenomenon first observed in response to influenza, it has since been found to be common across diverse taxa (Francis, 1953; Welsh and Fujinami, 2007). Original antigenic sin describes the shift in the immunodominance hierarchy that occurs when prior exposure to cross-reactive antigens alters and inhibits subsequent immune response to related antigens, either in new infections or through vaccination (Brehm et al., 2002). Both humoral and cellular responses can be plagued by such misdirected or inappropriate heterotypic immunity (Welsh and Fujinami, 2007). The phenomenon is most severe when the cross-reactive antigenic responses are immunodominant as is the case with both cellular and humoral DENV immunity. Thus, for DENV, not only does original antigenic sin appear to play an important role in the more severe DENV pathologies but it also creates a potential vaccine safety issue as a lack of protective tetravalent vaccine-induced immunity could prime vaccinees for DHF upon subsequent infection (Durbin and Whitehead, 2011; Murphy and Whitehead, 2011; Schmitz et al., 2011; Heinz and Stiasny, 2012).

Concerns for dengue vaccination priming individuals for severe disease via immune enhancement have necessitated that dengue vaccines must produce balanced, tetravalent, and protective immunity, optimally derived from a single dose vaccination providing long-lasting protection (Miller, 2010; Murphy and Whitehead, 2011; Heinz and Stiasny, 2012). Inducing balanced and protective immunity has been the biggest hurdle in DENV vaccination (Sun etal., 2009; Morrison et al., 2010; Durbin and Whitehead, 2011). Tetravalent live-attenuated vaccines are susceptible to replication interference between different vaccine viruses (Guy et al., 2009) requiring multiple boosts spread over 6-12 months (Sin Leo et al., 2012). Nevertheless, multiple live-attenuated DENV vaccines are advancing in human clinical trials and promising results suggest potential licensure this decade (Durbin and Whitehead, 2009; Guy et al., 2011; Heinz and Stiasny, 2012).

We have developed a second generation flavivirus vaccine platform attempting to address some of the potential obstacles to DENV vaccine development. The DNA vaccine platform consists of an expression plasmid containing only the envelope (E, the primary protective antigen) and premembrane (prM) structural protein genes. Upon uptake by host cells the structural genes are transcribed and translated and the proteins self-assemble into virus-like particles (VLPs) presenting authentic E proteins (Chang et al., 2001, 2003). Our wild-type (WT) flavivirus DNA vaccines are demonstrably safe, immunogenic, and protective against Japanese encephalitis, West Nile, and dengue viruses in both non-human animals (Davis et al., 2001; Chang et al., 2003, 2007; Hughes et al., 2012b) and in humans (Martin et al., 2007). DNA vaccines stimulate strong CD4+ T cell responses as do inactivated and subunit vaccines and yet also strongly stimulate CD8+ $\mathrm{T}$ cell responses similar to live-attenuated vaccines (Laylor et al., 1999; Martin et al., 2007). Because VLPs lack infectious RNA and are non-replicating, multivalent DNA vaccines are less susceptible to replication interference than live-attenuated vaccines (Petersen and Roehrig, 2007; Guy et al., 2009). Most importantly, DNA vaccines can be readily manipulated and engineered to prime specific epitopes and redirect immunity. This sculpted immune memory priming can redirect subsequent vaccine boosts or natural exposure toward protective, DENV serotype-specific epitopes increasing both vaccine safety and efficacy (Miller, 2010; Nara et al., 2011; Schmitz et al., 2011; Hughes et al., 2012a).

In this work we introduce substitutions into two distinct E protein antigenic regions of a WT DENV-1 DNA vaccine (pVD1-WT) ablating cross-reactive, immunodominant, weakly or non-neutralizing B cell epitopes (Crill et al., 2009) associated with immune enhancement, to limit the ability of this crossreactivity reduced vaccine ( $\mathrm{pVD} 1-\mathrm{CRR})$ to potentiate $\mathrm{DHF}$ via ADE. We extend upon previous proof in principle work with DENV-2 (Chang et al., 2003; Hughes et al., 2012a,b) to test pVD1CRR safety and efficacy via active vaccination of AG129 mice. 
Both pVD1-WT and pVD1-CRR were equally protective against lethal, homologous DENV-1 challenge. pVD1-CRR vaccinated mice produced significantly less antibody recognizing immunodominant enhancing epitopes, exhibited significantly increased serotype-specific and protective immunity in response to heterologous DENV-2 challenge, and were less susceptible to lethally enhanced heterologous DENV-2 DHF-like disease. These results provide new insights into sculpting humoral immunity that should advance the development of DENV vaccines and our understanding of the mechanisms of their protective efficacy.

\section{MATERIALS AND METHODS

VACCINE CONSTRUCTION, CHARACTERIZATION, AND PREPARATION

The construction of pVD1-WT and pVD1-CRR vaccine plasmids was the same as for DENV-2 DNA vaccine plasmids that have been previously described in detail (Chang et al., 2003; Crill et al., 2009). Briefly, pVD1-WT was constructed using DENV-1 strain (BC94/95) for cDNA cloning of prM and 80\% DENV-1 E protein with the C-terminal $20 \%$ of E replaced with the homologous region of Japanese encephalitis virus (JEV). Eighty percent DENV-1 E corresponds to the ectodomain and the C-terminal $20 \%$ corresponds to the helical stems (E-H1, E-H2) and transmembrane domain (TMD) anchor helices (E-T1, E-T2; Zhang et al., 2003; Lin et al., 2011). The pVD1-WT and pVD1-CRR vaccines utilized in this study also contained a potent CD4+ T cell epitope identified in West Nile virus (WNV) and located in the TMD. The inclusion of this epitope into our DENV-2 DNA vaccine (pVD2) and into pVD2-CRR was shown to boost the neutralizing antibody responses of vaccinated mice about twofold. The identification, construction, and characterization of this epitope has recently been described in detail (Hughes et al., 2012b). Introduction of the WNV TMD CD4+ epitope into pVD1 required introducing four amino acid substitutions (V474I, A484T, T488V, and V493L) into the C-terminal 20\% JEV E sequence of pVD1.

pVD1-CRR was constructed using the primers listed in Table 1 and pVD1-WT as the DNA template with Stratagene Quick Change multi, site-directed mutagenesis kit (Stratagene, La Jolla, CA) following the manufacturer's recommended protocols. Structural gene regions and regulatory elements of all plasmids were sequenced entirely upon identification of the correct mutation.

\section{ETHICS STATEMENT}

All animal experiments were approved by the IACUC at the US Centers for Disease Control and Prevention (CDC), Division of Vector-borne Disease (DVBD); an AAALAC certified facility. Protocols followed the US National Guidelines defined in the Guide for the Care and Use of Laboratory Animals, 8th edition, 2011, National Academy Press.

\section{MICE: PROTECTIVE EFFICACY}

Type I/type II IFN receptor-knock-out mice (AG129) were obtained from the DVBD, CDC colony. Six-week-old AG129 mice (DVBD, $n=26$ ) were immunized intramuscularly (i.m.) with $100 \mu \mathrm{g}$ of $\mathrm{pVD} 1-\mathrm{WT}$ or pVD1-CRR vaccine ( $50 \mu \mathrm{g}$ in each thigh) at 0,4 , and 8 weeks and with naïve age-matched controls $(n=8)$, were challenged at 12 weeks intraperitoneally (i.p.) with $1.1 \times 10^{5}$ focus forming units (FFU) of DENV-1 Mochizuki strain. Based upon results from previous dose-titration experiments four animals from each vaccine treatment and two naïve mice were $a$ priori selected for sacrifice 3 and 8 days post challenge (DPC). Sick animals reaching predetermined morbidity endpoints were anesthetized, total blood was collected via cardiac puncture, euthanized and carcasses were frozen at $-80^{\circ} \mathrm{C}$ for subsequent analysis.

\section{MICE: VACCINE SAFETY}

Six-week-old AG129 mice (DVBD, $n=26$ ) were immunized with $100 \mu \mathrm{g}$ of either $\mathrm{pVD} 1-\mathrm{WT}$ or $\mathrm{pVD} 1-\mathrm{CRR}$ vaccine $(50 \mu \mathrm{g}$ in each thigh, i.m. ) at 0 weeks and with age-matched naïve mice $(n=10)$ were i.p. challenged at 12 weeks with $4.2 \times 10^{5}$ FFU of DENV2 S221 (gift from S. Shresta; Zellweger et al., 2010). Four mice from each vaccine treatment and two naïve mice were a priori selected for sacrifice 3 DPC. Sick animals reaching predetermined morbidity endpoints were anesthetized, total blood was collected via cardiac puncture, euthanized, and necropsy was performed with livers collected and stored in 10\% formalin for histology.

\section{HISTOLOGY}

Liver tissue sections were prepared, stained, and analyzed by Todd Bass at the Colorado State University (CSU) College of Veterinary Medicine and Biomedical Sciences Histology Laboratories. Hematoxylin and Eosin (H\&E) staining and immunohistochemistry slides were interpreted by Dr. Barbara Powers at CSU and Dr. Nord Zeidner (CDC) assisted with H\&E slide interpretation and

Table 1 | Nucleotide sequences of mutagenic primers.

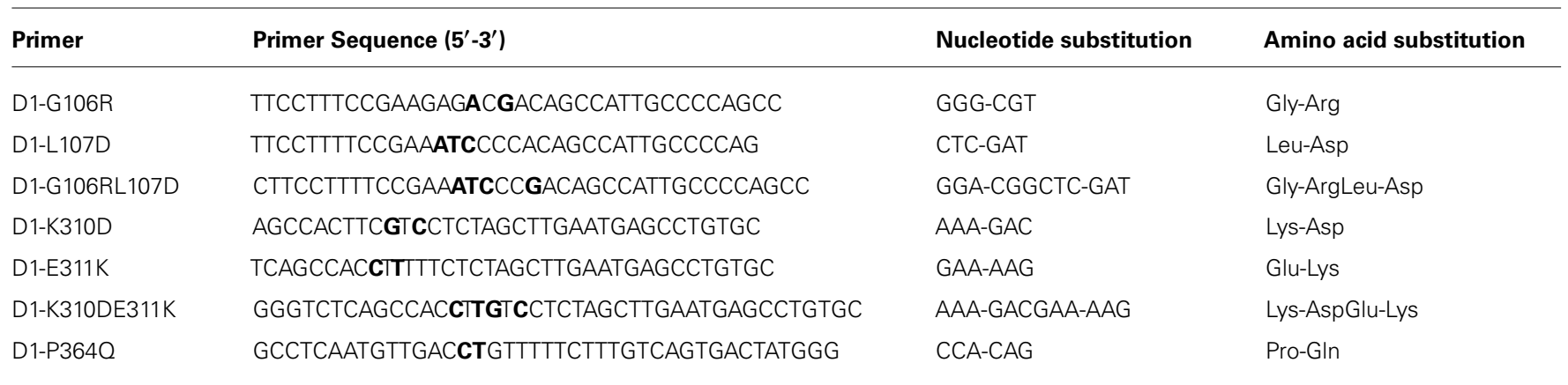

Mutated nucleotides are shown in bold. 
photography. IHC utilized Ventana Medical Systems ultraView Universal Alkaline Phosphatase Red Detection Kit following the manufacturer's recommended protocols and a Benchmark Ultra automated system. Pretreatment was with protease II for $6 \mathrm{~min}$, rabbit anti DENV-2 NS1 serum, generated at the CDC, was used at a 1:20 dilution, and anti-rabbit secondary antibody was applied for $12 \mathrm{~min}$.

\section{CHARACTERIZATION OF pVD1-CROSS-REACTIVITY REDUCED PLASMID VACCINE EXPRESSED VIRUS-LIKE PARTICLE ANTIGEN}

Antigen-capture ELISA (Ag-ELISA) was used to characterize VLP antigen following previously described protocols (Crill et al., 2009). Briefly, VLP antigen was collected from mutagenized and WT pVD1 transformed COS-1 cells. Secreted antigen was captured in the inner 60 wells of Immulon II HB flat-bottom 96-well plates (Dynatech Industries, Inc., Chantilly, VA) with polyclonal rabbit anti-DENV-1 WT VLP sera, incubated overnight at $4^{\circ} \mathrm{C}$, and wells were blocked with $300 \mu \mathrm{l}$ of StartBlock blocking buffer (Pierce, Rockford, IL, USA) according to the manufacturer's recommended protocol. Antigen was diluted twofold in PBS, incubated for $2 \mathrm{~h}$ at $37^{\circ} \mathrm{C}$ and detected with murine hyper-immune ascitic fluid (MHIAF) specific for DENV-2 diluted in 5\% milk/PBS. MHIAF was detected using horseradish peroxidase (HRP) conjugated goat anti-mouse IgG (Jackson ImmunoResearch, Westgrove, $\mathrm{PA}$ ) in $5 \%$ milk/PBS and incubated for $1 \mathrm{~h}$ at $37^{\circ} \mathrm{C}$. Bound conjugate was detected with 3,3'5,5' -tetramethylbenzidine substrate (TMB; Neogen Corp., Lexington, KY), the reaction was stopped with $2 \mathrm{~N} \mathrm{H}_{2} \mathrm{SO}_{4}$ and measured at $A_{450}$ using a Synergy HT Multi-Detection Microplate Reader (Bio-Tek Instruments, Inc., Winooski, VA). WT and mutant antigens were screened against the MAb panel using the same ELISA protocol as above with the exception that twofold dilutions of the specific MAb replaced the anti-DENV-2 MHIAF and antigens were used at a single standardized concentration producing an optical density (OD) of 1.0. Standardized concentrations of WT and mutant VLP antigens were analyzed in Ag-ELISA to determine MAb endpoint reactivities.

\section{MONOCLONAL ANTIBODIES}

MAbs 4G2, 6B6C-1, 4A1B-9, 1B7, D3-5C9-1, 1A1D-2, 9D12, 9A3D-8, 3H5, and D2-1F1-3 were obtained from hybridomas in the collection of the Arboviral Diseases Branch, Division of Vector-borne Diseases, Centers for Disease Control and Prevention. Many of these MAbs originated from the work of John Roehrig (Roehrig et al., 1998), 4G2, 5H3, 1B7, D3-5C9-1, 9D12, $3 \mathrm{H} 5$, and D2-1F1-3 hybridomas were originally obtained by the CDC from the Walter Reed Army Institute (Henchal et al., 1985). MAbs 23-1, 23-2, and 5-2 were provided by Dr. L.-K. Chen of Tzu Chi University, Hualien, Taiwan. MAbs 10-D35A, 20-783-745014, and MDVP-55A are commercially available and were purchased from Fitzgerald Industries International, GenWay Biotech Inc., and Immunology Consultants Laboratory Inc. respectively.

\section{EPITOPE-SPECIFIC IgG ELISA}

We used DENV-1 and DENV-2 epitope-specific IgG ELISA (ESELISA) to determine total DENV-1 or DENV-2 IgG endpoint titers and to determine percent DENV-1 or DENV-2 EDII
non-EDII ${ }_{\mathrm{FP}}$ EDIII $_{\mathrm{CR}}$ epitope-specific IgG. The DENV-2 ES-ELISA has been previously described (Crill et al., 2009; Hughes et al., 2012a) and the DENV-1 ES-ELISA was similarly used with a few modifications. Briefly, Rabbit anti-pVD1 or pVD2 sera was coated onto plates overnight at $4^{\circ} \mathrm{C}$. Standardized concentrations of WT and CRR antigens were captured, AG129 mouse serum was diluted two- to fourfold down the plate and detected with goat anti-mouse IgG (Jackson ImmunoResearch). The DENV1 epitope-specific knock-out antigens utilized are described in Table 2. $\mathrm{OD}_{450}$ values were modeled as non-linear functions of the $\log _{10}$ serum dilutions using a non-linear sigmoidal dose-response (variable slope) regression in Graph Pad Prism version 4.0 (GraphPad Software, Inc. La Jolla, CA) and endpoint titrations were determined as the titer where the $\mathrm{OD}_{450}$ value equaled two-times the $\mathrm{OD}_{450}$ value of the test serum reacted against normal COS-1 antigen.

E protein structural domain II fusion peptide $\left(\mathrm{EDII}_{\mathrm{FP}}\right)$ and non-EDII $I_{F P}$ EDIII cross-reactive $\left(\right.$ EDII $_{F P}$ EDIII $\left._{C R}\right)$ epitopespecific IgG percentages were calculated as previously described (Crill et al., 2009; Hughes et al., 2012a) with minor modifications. The DENV-1 epitope-specific IgG percentages for all vaccinated mice were calculated by dividing the IgG endpoint titer obtained with each knock-out antigen $\left(\mathrm{EDII}_{\mathrm{FP}}\right.$ or $\left.\mathrm{EDII}_{\mathrm{FP}} \mathrm{EDIII}_{\mathrm{CR}}\right)$ by the endpoint titer obtained with pVD1-WT antigen on the same sera, subtracting this value from 1.0, and multiplying by 100 . Specifically, EDII $\mathrm{FP}_{\mathrm{F}}$ epitope-specific percentages were calculated as $100 \times\left[1.0-\left(D E N V-1\right.\right.$ EDII $_{\mathrm{FP}}$ antigen endpoint/ DENV-1 WT antigen endpoint)]. Non-EDII ${ }_{F P} E D I I I_{C R}$ IgG proportions were calculated as $100^{\star}$ (endpoint $\mathrm{EDII}_{\mathrm{FP}} \mathrm{EDIII}_{\mathrm{CR}} /$ endpoint WT) for WT vaccinated mice and because $\mathrm{pVD1-CRR} \mathrm{vaccinated} \mathrm{mouse}$ sera contain antibodies that do not recognize some WT antigen epitopes (WT antigen acts as the knock-out antigen) but recognize the modified epitopes of the CRR antigens, the pVD1-CRR antigen $\left(\right.$ EDII $\left._{\mathrm{FP}} \mathrm{EDIII}_{\mathrm{CR}}\right)$ replaced pVD1-WT antigen to determine $100 \%$ E reactivity for $\mathrm{pVD1-CRR}$ vaccinated mice and similarly, the nonEDII $_{\mathrm{FP}}$ EDIII $_{\mathrm{CR}}$ IgG proportion for $\mathrm{pVD1-CRR} \mathrm{immunized} \mathrm{mice}$ was calculated as $100^{\star}(\mathrm{DENV}-1 \mathrm{WT}$ antigen endpoint/ DENV1 EDII $_{\mathrm{FP}}$ EDIII $_{\mathrm{CR}}$ antigen endpoint). DENV-2 epitope-specific IgG populations were similarly determined but used the previously described DENV-2 EDII $\mathrm{FP}_{\mathrm{F}}$ and $\mathrm{EDII}_{\mathrm{FP}} \mathrm{EDIII}_{\mathrm{CR}}$ knock-out antigens to determine endpoints (Crill et al., 2009; Hughes et al., 2012a) for both pVD1-WT and pVD1-CRR vaccinated sera. In cases where the endpoint titer determined with a mutant antigen was the same or greater than the endpoint titer obtained with the cognate antigen we interpreted these cases as undetectable levels of antibody recognizing the epitope of interest and the percent of IgG was conservatively set at $1.0 \%$. Calculated $\mathrm{EDII}_{\mathrm{FP}} \mathrm{IgG}$ end-

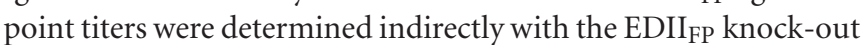
and WT antigens by multiplying the total IgG endpoint titer by the percent $\mathrm{EDII}_{\mathrm{FP}} \mathrm{IgG}$.

\section{EPITOPE-BLOCKING ELISA}

Epitope blocking ELISA was utilized to determine the vaccinated mouse response to well-characterized murine MAb epitopes. This ELISA was set up similar to ES-ELISA in that plates were coated overnight at $4^{\circ} \mathrm{C}$ with rabbit anti-DENV-2 serum, blocked with StartBlock (Pierce), dried and WT DENV-2 VLP antigen was 


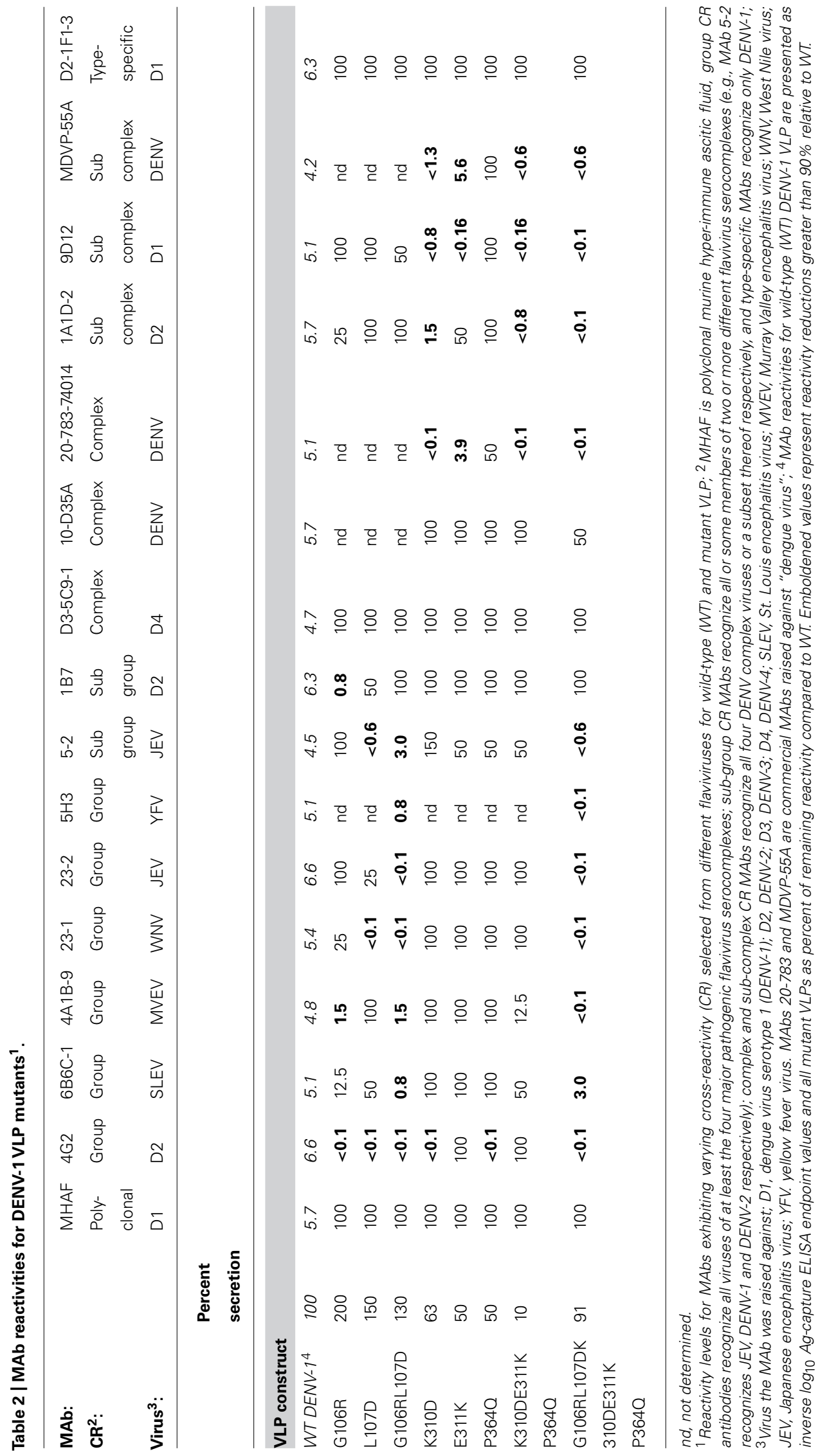


captured $1 \mathrm{~h}$ at $37^{\circ} \mathrm{C}$. After washing, pVD1-WT or pVD1-CRR vaccinated, DENV-2 challenged mouse serum (3 DPC, pooled for each vaccine treatment due to limited availability of serum) was diluted 1:40 in wash buffer and incubated $1 \mathrm{hr}$ at $37^{\circ} \mathrm{C}$. Following pooled serum incubation and wash, $0.5 \mu \mathrm{g}$ of MAb conjugated with HRP was added to each well and incubated for $1 \mathrm{~h}$ at $37^{\circ} \mathrm{C}$ to compete with the already bound antibody from vaccinated mouse serum for WT DENV-2 VLP antigen. Bound conjugate was detected with TMB substrate and plates incubated for $10 \mathrm{~min}$ prior to being stopped with $\mathrm{H}_{2} \mathrm{SO}_{4}$ and ODs were determined as $\mathrm{OD}=\mathrm{OD}$ of $\mathrm{A}_{450}-\mathrm{OD}$ of $\mathrm{A}_{630}$. Percent blocking was determined by comparison to replicate wells with $0.5 \mu \mathrm{g}$ HRP labeled MAb competing against pre-adsorbed normal mouse serum using the following calculation, $100-100^{*}[(\mathrm{OD}$ of vaccinated serum on DENV-2 Ag-OD of vaccinated serum on normal Ag)/OD of normal serum on DENV-2 Ag-OD of normal serum on normal Ag)].

\section{IN VITRO ANTIBODY-DEPENDENT ENHANCEMENT}

Heat inactivated pVD1-WT and pVD1-CRR vaccinated mouse sera (12 weeks post vaccination) were pooled into four groups for each vaccine treatment, diluted, and titrated. Virus (DENV2 16681) was added to each dilution and incubated for $1 \mathrm{~h}$ at $37^{\circ} \mathrm{C}$. K562 cells (MOI $=0.5$ ) were added to the antibodyvirus complexes and incubated $2 \mathrm{~h}$. After infection, cells were centrifuged, supernatants removed, resuspended in RPMI media (10\% FBS) and plated. DENV infection alone was used as virus control.

\section{FOCUS REDUCTION MICRO-NEUTRALIZATION ASSAY}

Focus reduction micro-neutralization assay (FR $\mu \mathrm{NT})$ assay was utilized as previously described (Crill et al., 2009) with few modifications. Vaccinated mouse sera were diluted 1:10, heat inactivated, titrated twofold to the volume of $40 \mu \mathrm{L}$, and 320 virus FFU/40 $\mu \mathrm{L}$ (DENV-1 56BC94/95, DENV-2 16681, DENV3 116RC1396, or DENV-4 130) was added to each dilution. FR $\mu$ NT titers were calculated for each virus relative to a back titration. Exact 50\% of FR $\mu$ NT titers were modeled using Graph Pad Prism version 4 sigmoidal dose response (variable slope) non-linear regression. Values are the average of two independent replicates.

\section{VIREMIA AND BRAIN VIRUS TITERS}

Viremia was determined in a similar antigen-focus assay as described for FR $\mu$ NT except that no virus was added. Cells were incubated, overlaid, acetone fixed, immunostained, and counted as described for FR $\mu$ NT. Virus brain titers were determined from previously frozen mouse carcasses. Brain tissue was aspirated with a $3 \mathrm{~mL}$ syringe and 18 gage needle, weighed, and resuspended in $200 \mathrm{uL}$ BA-1 media. Fifty microliters was used in the FR $\mu$ NT as described. FFU/g of tissue was back calculated from the aspirated brain mass.

\section{STATISTICAL ANALYSIS}

Non-parametric analyses used the Mann-Whitney $U$ test and paired analyses utilized the paired $t$-test. All statistical analyses were performed using GraphPad Prism 4.02.

\section{RESULTS}

\section{VACCINE CONSTRUCTION}

Based upon previously published and unpublished results with DENV and other flaviviruses (Crill and Chang, 2004; SukupolviPetty et al., 2007; Gromowski et al., 2008; Crill et al., 2009; Hughes etal., 2012a), we introduced specific substitutions into crossreactive $B$ cell epitopes of the envelope protein of a DENV-1 prM/E expression plasmid (Chang et al., 2003) to generate a DENV-1 DNA vaccine candidate with reduced ability to induce the crossreactive antibodies that can be associated with ADE. The DENV-1 WT DNA vaccine (pVD1-WT) utilized as the template for the CRR vaccine (pVD1-CRR) in this study was based upon previous studies with DENV-2 vaccines and contains a potent CD4+ epitope identified in WNV and demonstrated to increase the immunogenicity of DENV-2 DNA vaccines (Hughes et al., 2012b). We introduced substitutions into two important cross-reactive regions of the DENV E protein, the highly conserved fusion peptide of structural domain II $\left(\mathrm{EDII}_{\mathrm{FP}}\right)$ and the A-strand and DE loop of domain III (EDIII; Figure 1).

EDII $_{F P}$ contains multiple overlapping immunodominant B cell epitopes inducing broadly cross-reactive, weakly or nonneutralizing antibodies associated with antibody-enhanced severe DENV disease in both mice and humans (Stiasny et al., 2006; Lai etal., 2008; Crill etal., 2009; Balsitis etal., 2010; Beltramello et al., 2010; Zellweger et al., 2010). EDIII contains two well-characterized overlapping antigenic regions, one stimulating DENV complex cross-reactive antibodies varying in their neutralizing capabilities (Roehrig et al., 1998; Sukupolvi-Petty et al., 2007; Gromowski et al., 2008; Cockburn et al., 2012) and the other stimulating DENV serotype-specific, potently neutralizing antibodies associated with DENV serotype-specific immunity (Gromowski and Barrett, 2007; Sukupolvi-Petty et al., 2007; Crill et al., 2009; Beltramello et al., 2010). We examined multiple substitutions at EDII $_{\mathrm{FP}}$ residues G106 and L107 and at K310, E311, and P364 in the cross-reactive antigenic region of EDIII (EDIII ${ }_{\mathrm{CR}}$; Figure 1). Final individual substitutions at these five residues were selected based upon their influence on in vitro VLP secretion and their effect on the reactivities of a panel of MAbs (Table 2).

EDII $_{\mathrm{FP}}$ substitutions tended to increase VLP secretion and knocked-out the reactivity of flavivirus group and sub-group cross-reactive MAbs. EDIII ${ }_{\mathrm{CR}}$ substitutions tended to reduce VLP secretion relative to $\mathrm{WT}$ and ablated the reactivity predominately of DENV sub-complex cross-reactive MAbs. EDIII ${ }_{\mathrm{CR}}$ substitutions were specifically selected not to interfere with the binding of potently neutralizing EDIII lateral ridge serotype-specific MAbs. Although human MAbs recognizing similar epitopes have been identified, a growing body of evidence suggests they may not comprise the main antigenic targets of the human neutralizing antibody response (Beltramello et al., 2010; Dejnirattisai et al., 2010; de Alwis et al., 2011; Wahala et al., 2012). The final pVD1CRR plasmid, containing substitutions at all five of the sites across both $\mathrm{EDII}_{\mathrm{FP}}$ and $\mathrm{EDIII}_{\mathrm{CR}}$ antigenic regions (Figure 1), knocked-out or reduced the reactivity to below detectable levels of 11 cross-reactive monoclonal antibodies in our panel (Table 2). The cross-reactive MAbs whose reactivities were not significantly reduced were $1 \mathrm{~B} 7$, a sub-group cross-reactive $\mathrm{MAb}$ that neutralizes all four DENV serotypes, and 10-D35A and D3-5C9-1, 

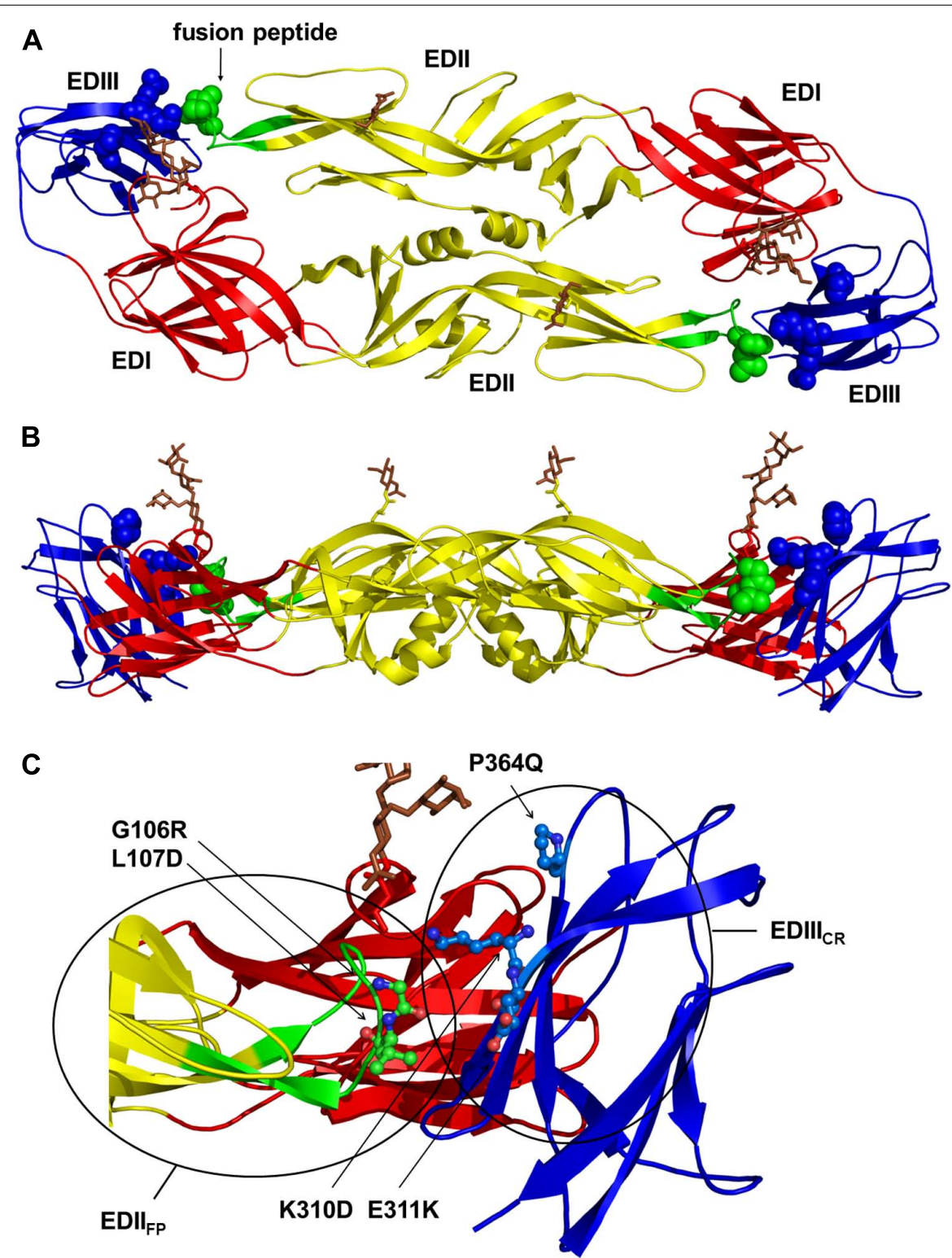

FIGURE 1 | Structural locations of the pVD1-CRR envelope (E) protein cross-reactive epitope knock out substitutions. The locations of the DENV-1 E substitutions introduced to construct PVD1-CRR mapped on the crystal structure of the homologous DENV-2 E protein dimer (Modis etal., 2003). (A) Locations of pVD1-CRR substitutions (spheres) on a ribbon diagram of the mature $\mathrm{E}$ dimer as it appears looking straight down toward the virion surface. The three structural domains are labeled and colored red for $\mathrm{E}$ domain I (EDI), yellow for EDII, and blue for EDIII. The EDII fusion peptide $\left(E D \|_{F P}\right)$ is also labeled and colored green. The glycans in EDI (N67) and EDII
(N153) are depicted as stick representations and colored brown. (B) Side view of the $\mathrm{E}$ protein dimer with all colorations the same as in panel $\mathrm{A}$. (C) Close up of the EDII FP from one monomer and EDIII region of the other at the dimer interface, as it appears in panel B. The side chains of two residues in the EDIIFP and three residues in EDIII where CRR substitutions were introduced are depicted as ball and stick representations and labeled with the introduced substitutions. Structural domains are colored as in panel A and the two antigenic regions $E D I_{F P}$ and $E D I I I_{C R}$ are noted with circles roughly representing the size of an IgG footprint binding to these regions. weakly (for DENV-2 only) and non-neutralizing DENV complex cross-reactive MAbs respectively.

\section{PROTECTIVE EFFICACY}

Both pVD1-WT and pVD1-CRR vaccines induce similarly high titer IgG and protect mice from lethal DENV-1 challenge

To test for vaccine protective efficacy we compared WT and CRR DENV-1 DNA vaccines by immunizing groups of AG129 mice
( $n=26$ ) and subsequently challenging with a lethal dose of homologous DENV-1 (Mochizuki strain). AG129 type I/type II IFN receptor knock-out mice have impaired neutralizing antibody responses (Schijns et al., 1994) and as expected our DNA vaccines were not as immunogenic in these mice as in immunocompetent mouse strains (data not shown). We therefore altered our standard immunization schedule from a single $100 \mu \mathrm{g}$ boost 4 weeks following primary vaccination $(100 \mu \mathrm{g})$ to two 
$100 \mu \mathrm{g}$ boosts at 4 and 8 weeks. This schedule elicited IgG immune responses approaching the magnitude of those previously observed in other mouse strains with the two dose schedule (Chang et al., 2003; Hughes et al., 2012a,b). Mice were challenged at 12 weeks. Prior to challenge, mouse sera was collected to measure vaccine-induced humoral immune responses. We used ES-ELISA (Crill et al., 2009) to determine total DENV-1 IgG and

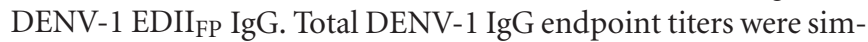
ilar between the two vaccines and averaged $7.7 \times 10^{4} \pm 1.5 \times 10^{4}$ and $7.5 \times 10^{4} \pm 1.8 \times 10^{4}$ for $\mathrm{pVD} 1-\mathrm{WT}$ and $\mathrm{pVD} 1-\mathrm{CRR}$ immunized mice respectively ( $p=0.524$; Figure 2A). However, pVD1-CRR immunized mice had significantly lower proportions of EDII $_{\mathrm{FP}}$ IgG than did pVD1-WT immunized mice, averaging $2.1 \pm 0.96$ and $33 \pm 6.4 \%$ of the total IgG response respectively ( $p=0.0005$, Figure 2B). Only two pVD1-CRR immunized mice had measurable EDII FP $_{\text {IgG }}(4.8$ and $22 \%)$ with the remaining mice being below detectable levels (conservatively set to $1.0 \%$ for statistical analyses and $\mathrm{EDII}_{\mathrm{FP}}$ IgG titer calculations). The proportion of $\mathrm{EDII}_{\mathrm{FP}}$ IgG for WT immunized mice was large and variable, ranging from 0.8 to $73 \%$, similar to that observed in DENV infected humans (Stiasny et al., 2006; Crill et al., 2009;

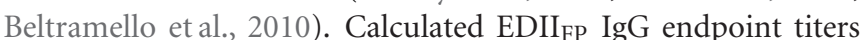
averaged $2.0 \times 10^{4} \pm 4.6 \times 10^{3}$ and $1.1 \times 10^{3} \pm 3.4 \times 10^{2}$ for WT and CRR immunized mice respectively $(p=0.0001$, Figure $2 \mathrm{C})$. Fifty percent neutralization $\left(\mathrm{Nt}_{50}\right)$ titers, measured by FR $\mu$ NT, averaged 91.1 and 48.8 for pVD1-WT and pVD1CRR vaccinated mice respectively $(p=0.0047$; Figure $2 \mathrm{D})$. The lower $\mathrm{Nt}_{50}$ titer for $\mathrm{pVD1-CRR}$ immunized mice was likely due to reduced induction of $\mathrm{EDIII}_{\mathrm{CR}}$ antibodies recognizing epitopes similar to those of neutralizing MAbs 1A1D-2 and 9D12 that lost all measurable reactivity for the pVD1-CRR tissue culture derived VLPs (Table 1). These results suggest that targeted
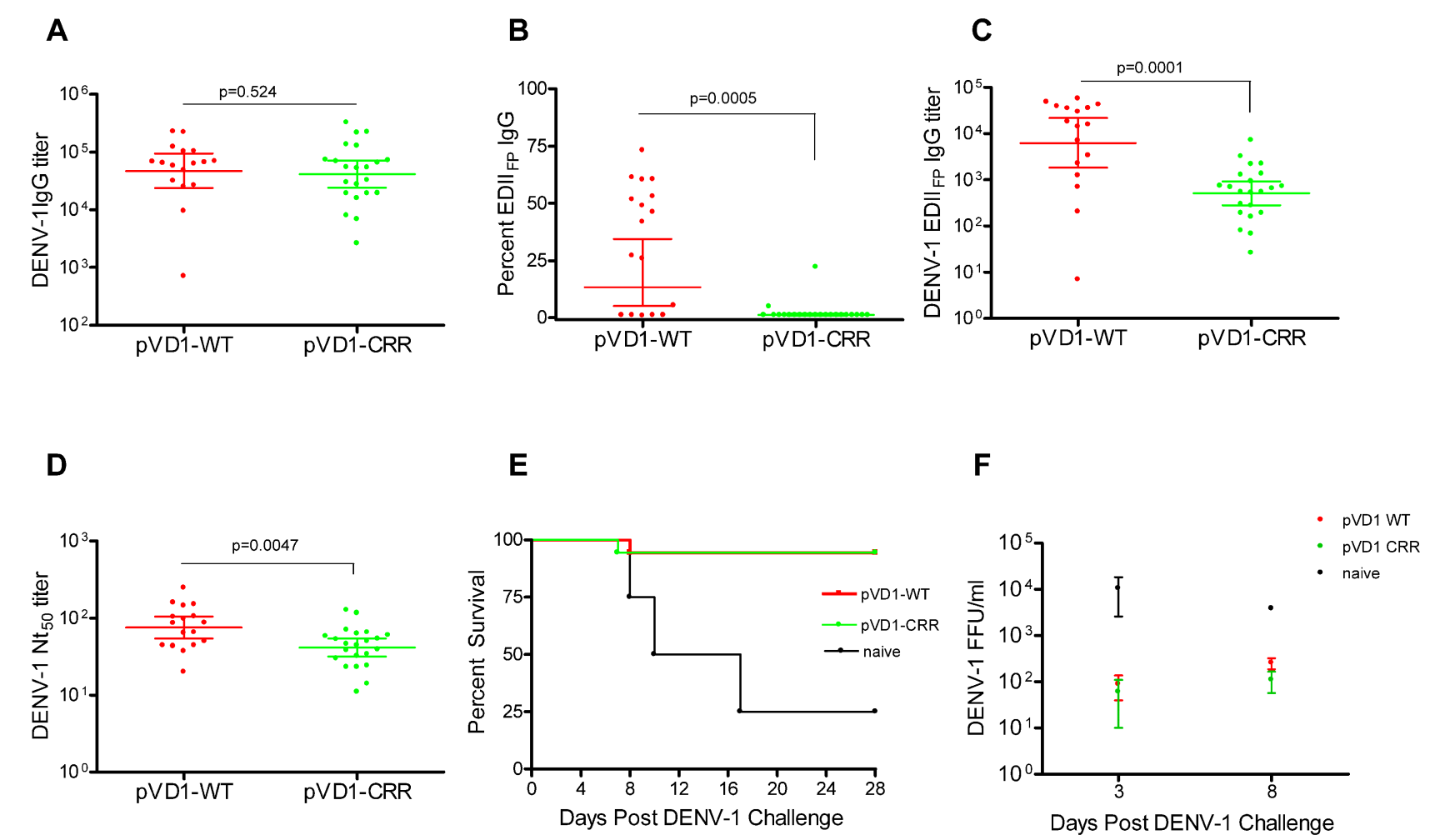

FIGURE 2 | Protective Efficacy: Both pVD1-WT and pVD1-CRR vaccines protect AG129 mice against lethal DENV-1 challenge. (A-D) 12 week post vaccination immunogenicity of $A G 129$ mice immunized (i.m.) with $100 \mu \mathrm{g}$ of pVD1-WT or pVD1-CRR vaccines at 0, 4, and 8 weeks. Geometric means and $95 \%$ confidence intervals are depicted unless otherwise noted. All endpoint titers were $\log _{10}$ transformed and statistical significance was determined using the Mann-Whitney $U$ test to account for non-normality of some transformed data. Panels B and $C$ both used a one-tailed test since there was an a priori expectation that pVD1-CRR immunized mice would exhibit reduced EDII FP recognizing IgG. (A) DENV-1 total IgG endpoint titers 12 weeks post immunization. (B) Percent of total DENV-1 IgG recognizing immunodominant EDII ${ }_{F P}$ epitopes, arithmetic means and $95 \% \mathrm{Cl}$ depicted. (C) Calculated DENV-1 EDII FP IgG endpoint titers. (D) DENV-1 50\% antigen focus-reduction micro neutralization titers $\left(\mathrm{FR} \mu N T_{50}\right)$. A t test was utilized here as both data sets were normally distributed. (E) pVD1-WT and pVD1-CRR immunized AG129 mice were challenged with $1.1 \times 10^{5}$ FFU of DENV-1 (Mochizuki strain, i.p.) at 12 weeks. Kaplan-Meier survival curves are shown. Both pVD1-WT $(n=18)$ and pVD1-CRR ( $n=18$ ) vaccinated animals had the same survival $(94 \%)$, which was highly significant in comparison to naïve mice ( $n=4, p=0.0003$ ). (F) Four mice from each vaccine treatment (not included in the survival curves) were a priori scheduled to be euthanized 3 and 8 DPC. DENV-1 titers (FFU/mL) were $\log _{10}$ transformed and analyzed using two-way ANOVA; error bars represent standard error of the mean (SEM). Vaccine treatment was highly significant $(p<0.0001)$. Bonferroni post hoc tests indicated that viremia of each vaccinated group was significantly lower than for naive mice $(n=2) 3$ DPC ( $p<0.001)$, only pVD1-CRR vaccinated mouse viremia was significantly lower than naïve mice $(n=2) 8$ DPC $(p<0.01)$ and there was no difference between WT or CRR vaccinated mouse viremia 3 or 8 DPC. 
substitution within $\mathrm{EDII}_{\mathrm{FP}}$ can reduce the immunodominance of this region.

Age-matched naïve (18-week-old, $n=8)$, pVD1-WT and pVD1-CRR ( $n=26$ each) vaccinated animals were challenged with $1.1 \times 10^{5} \mathrm{FFU}$ of the mouse-brain adapted DENV-1 Mochizuki strain. This dose was $>100 \mathrm{LD}_{50}$ for 6-8-week-old mice (data not shown). All immunized mice except a single animal from each vaccine treatment were protected and survived challenge (94\% survival) which was highly significant in comparison to naïve mice (25\% survival, $p=0.0003$; Figure $2 \mathrm{E}$ ). Time to death of naïve mice ranged from 8 to $17 \mathrm{DPC}$ and averaged $11.7 \mathrm{DPC}$; the single vaccinated animals died 7 and 8 DPC for WT and CRR vaccine treatments respectively. Surviving mice showed no signs of sickness. Although DENV-1 Mochizuki is a mouse-brain adapted virus, we observed limited neurological symptoms such as paralysis in terminally sick mice and most exhibited hunched and ruffled posture, lethargy, and a lack of interest in food and water leading to weight loss prior to being euthanized. Four mice from each vaccine treatment and two naïve mice were scheduled a priori to be euthanized at $3 \mathrm{DPC}$, a time point prior to any outward sign of disease, and 8 DPC, a few days after the initial signs of morbidity and mortality. Vaccinated mice exhibited 100-fold and 10-fold lower viremia 3 and 8 DPC respectively, compared to naïve controls; yet there was no difference in mean viremic titers between $\mathrm{WT}$ or CRR vaccinated mice at either time point (Figure 2F). A two-way ANOVA found vaccine treatment to be highly significant $(p<0.0001)$ and to account for $68 \%$ of the variation. Bonferroni post hoc tests indicated that viremia of each vaccinated group was significantly lower than for naïve mice 3 DPC $(p<0.001)$ whereas only pVD1-CRR immunized mouse viremia was significantly lower than naïve mice $8 \mathrm{DPC}$ $(p<0.01)$.

Because DENV-1 Mochizuki strain is a mouse-brain adapted virus, we also determined virus titers of mouse-brain homogenates. Brains from both naïve and vaccinated mice were all negative 3 DPC ( $<50$ FFU/g brain tissue). By 8 DPC, the DENV-1 titer of naïve mouse-brain tissue was 100-1000 times greater than for vaccinated mice. Of note, we only had tissue from a single 8 DPC naïve mouse-brain precluding rigorous statistical analysis. Nevertheless, the single 8 DPC naive mouse-brain virus titer was $2.3 \times 10^{4} \mathrm{FFU} / \mathrm{g}$, geometric mean titers of WT and CRR vaccinated mice ( $n=4$ each) were 201 and $731 \mathrm{FFU} / \mathrm{g}$ of brain tissue respectively with three $\mathrm{WT}$ and two CRR vaccinated titers below the limits of assay detection ( $<50 \mathrm{FFU} / \mathrm{g}$ tissue). There was no difference in titer between WT and CRR vaccinated mouse-brains as determined by $t$-test or Mann-Whitney $U$ test (data not shown). A Wilcoxon signed-rank test comparing all eight vaccinated mousebrain titers to the $2.3 \times 10^{4}$ titer for the naïve mouse-brain tissue strongly rejected the null hypothesis of no difference $(p=0.0059)$, suggesting that there was less virus in vaccinated compared to naïve mouse brains 8 DPC.

Consistent with an anamnestic response to DENV-1 challenge, both WT and CRR immunized surviving mice exhibited similar order of magnitude increases in $\mathrm{Nt}_{50}$ titer $28 \mathrm{DPC}(\mathrm{GMT}=690$, 95\% CI 504-944 and =362, 95\% CI 218-601 for WT and CRR respectively). Together, these data suggest that pVD1-WT and pVD1-CRR vaccines are similarly immunogenic and able to induce protective immunity against lethal homologous DENV-1 challenge in the AG129 DENV vaccine model.

\section{VACCINE SAFETY: CROSS-REACTIVE REDUCED VACCINE REDIRECTS IMMUNITY FROM IMMUNODOMINANT PATHOLOGICAL TOWARD PROTECTIVE RESPONSES}

Groups of AG129 mice $(n=26)$ were immunized with a single $100 \mu \mathrm{g}$ dose of pVD1-WT or pVD1-CRR vaccine and subsequently challenged with a sub-lethal dose of heterologous DENV-2 S221 12 weeks post vaccination, the same timeline used for the homologous DENV-1 protective efficacy challenge above. DENV-2 S221 is a mouse adapted isolate of Taiwanese strain PLO46 (Shresta et al., 2006) capable of producing lethal hemorrhagic disease in AG129 mice - similar to that observed in human DHF - via ADE when sub-protective levels of DENV immune sera are passively transferred to mice subsequently challenged with non-lethal doses of DENV-2 S221 (Balsitis et al., 2010; Zellweger et al., 2010). We hypothesized that pVD1-WT immunized mice would be susceptible to antibody-enhanced DENV disease due to high levels of vaccine-induced cross-reactive antibodies and that upon heterologous challenge; antibodies recognizing these epitopes would be stimulated anamnestically. Conversely, pVD1-CRR vaccinated mice should lack such cross-reactive antibody priming and secondary responses, be less susceptible to antibody enhanced viral replication, and exhibit increased survival.

\section{DENV-1 CRR vaccinated mice have reduced levels of immunodominant EDII $I_{F P}$ IgG and reduced dengue disease mortality compared to DENV-1 WT vaccinated mice following DENV-2 challenge}

To assess if pVD1-WT and -CRR vaccines induce different antibody repertoires in vaccinated mice we collected 12-week post-vaccination sera and utilized ES-ELISA (Crill et al., 2009) to determine vaccine induced DENV-1 IgG titers and the proportions and titers of IgG recognizing EDII $_{\mathrm{FP}}$ epitopes (Figure 1). There was no difference in total DENV-1 IgG GMT between pVD1-WT $(3168,95 \%$ CI 1792-5600; $n=20)$ and pVD1-CRR immunized mice (1429, 95\% CI 621-3289, $n=23 ; p=0.128$, two-tailed Mann-Whitney $U$ test; Figure 3A). The percent of total DENV-1 IgG recognizing EDII ${ }_{\mathrm{FP}}$ epitopes averaged $17.7 \pm 4.6 \%$ and $2.56 \pm 1.2 \%$ for pVD1-WT and pVD1-CRR immunized mice respectively $(p=0.0009)$. Again, only two CRR immunized mice had detectable levels of $\mathrm{EDII}_{\mathrm{FP}} \operatorname{IgG}(9.4 \%$ and $21 \%)$ with all remaining mice having no measurable $\mathrm{EDII}_{\mathrm{FP}} \operatorname{IgG}(=1.0 \%$ for statistical analyses; Figure 3B). The calculated DENV-1 IgG GMT recognizing $\mathrm{EDII}_{\mathrm{FP}}$ epitopes was significantly lower for CRR (38.6, 95\% CI 19.4-76.9) than for WT immunized mice (272, 95\% CI 97.2-760, $p=0.0023$, one-tailed Mann-Whitney $U$ test; Figure 3C). These results again suggest that pVD1WT and pVD1-CRR vaccines are similarly immunogenic overall, but that the pVD1-CRR vaccine induces less IgG recognizing immunodominant EDII $_{\mathrm{FP}}$ epitopes.

Age-matched naïve $(n=10)$, pVD1-WT, and pVD1-CRR ( $n=26$ each) immunized mice were challenged (i.p.) with a sub-lethal dose $\left(4.2 \times 10^{5}\right.$ FFU) of DENV-2 S221 12 weeks following immunization for vaccine treatment groups. All naïve mice survived DENV-2 challenge, though they did exhibit signs of 


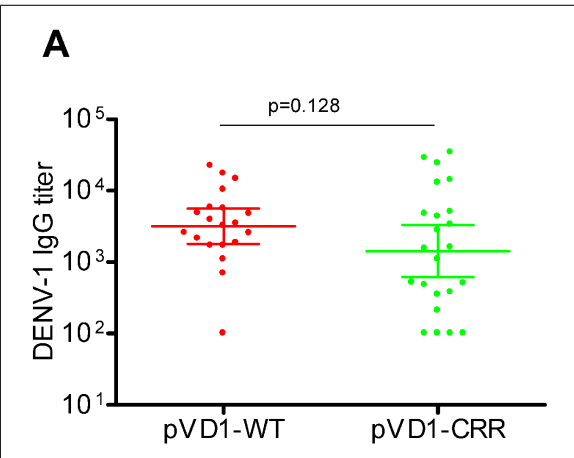

D

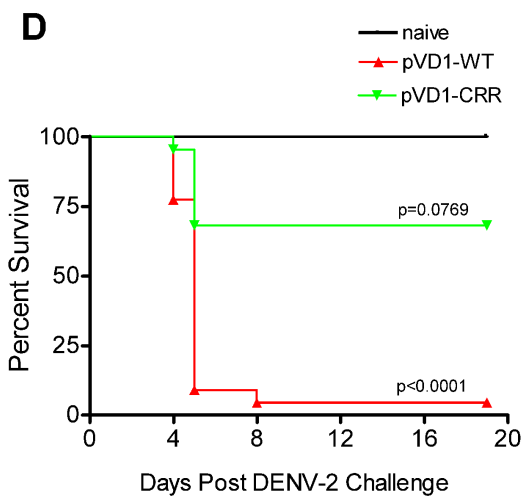

B

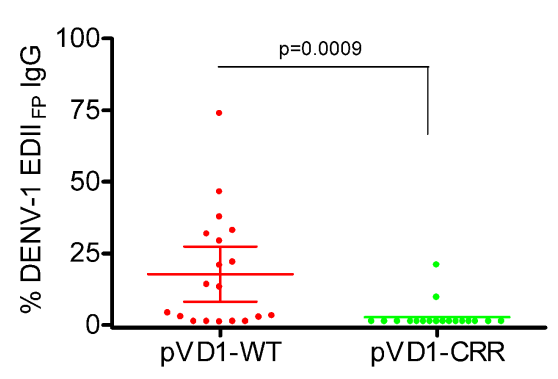

E

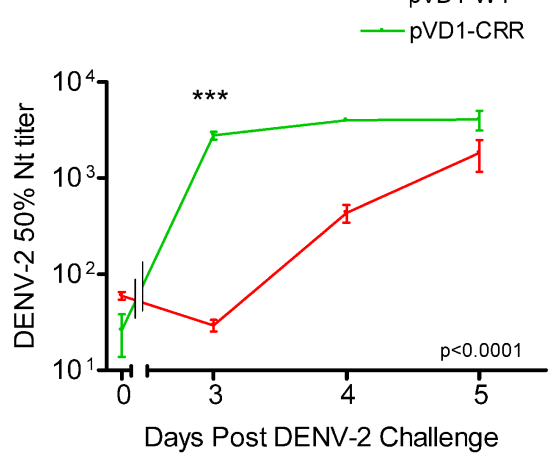

C

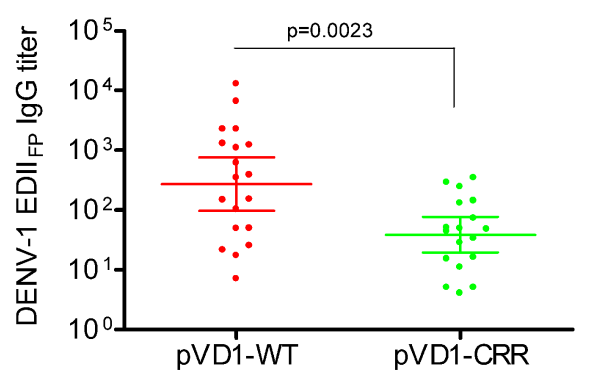

FIGURE 3 | DENV-1 CRR vaccine stimulates reduced levels of immunodominant cross-reactive EDII ${ }_{F P} \operatorname{IgG}$ and reduces enhanced DENV-2 mortality. (A-C) 12 week post vaccination immunogenicity of AG129 mice immunized (i.m.) with $100 \mu \mathrm{g}$ of pVD1-WT or pVD1-CRR vaccines. Geometric means and 95\% confidence intervals are depicted. Endpoint titers were $\log _{10}$ transformed and statistical significance was determined using the two-tailed Mann-Whitney $U$ test. Panels B and $C$ both used a one-tailed test since there was an a priori expectation that pVD1-CRR immunized mice would exhibit reduced EDII $\mathrm{FP}$ recognizing IgG. (A) DENV-1 total IgG endpoint titers 12 weeks post immunization. (B) Percent of total DENV-1 IgG recognizing immunodominant EDII ${ }_{F P}$ epitopes, arithmetic means and $95 \% \mathrm{Cl}$ depicted. (C) Calculated DENV-1 EDII FP IgG endpoint titers. (D) Survival of pVD1-WT and pVD1-CRR immunized AG129 mice following sub-lethal heterologous DENV-2 challenge. 12 weeks following vaccination, immunized mice $(n=22)$ or age matched naïve controls $(n=8)$ were challenged (i.p.) with $4.2 \times 10^{5}$ FFU of DENV-2 S221. Kaplan-Meier survival curves and $p$ values are shown. All naïve mice survived virus challenge with minimal signs of morbidity, pVD1-WT vaccinated mice suffered $95 \%$ mortality from enhanced DENV-2 disease ( $p<0.0001$, compared to naive). pVD1-CRR immunized mice exhibited $68 \%$ survival which did not differ from naïve mouse survival $(100 \%, p=0.0769)$ yet was significantly greater than pVD1-WT immunized mouse survival $(4.5 \%, p<0.0001)$. The Bonferroni multiple comparison adjusted $\alpha=0.017$. (E) pVD1-CRR vaccinated mice exhibited a rapid, large magnitude increase in DENV-2 neutralizing antibody titers following DENV-2 challenge. FR $\mu N_{50}$ titers determined with DENV-2 16681 virus on Vero cells, $\log _{10}$ transformed and the transformed data analyzed by two-way ANOVA ( $p<0.0001)$. Bonferroni post hoc test significance is depicted with asterisks, error bars represent SEM. 0, 3, 4, and 5 DPC $n=4,4,5$, and 9 for WT and 4, 4, 1, and 3 for CRR immunized mice respectively. (F) Viremia of pVD1-WT vaccinated mice increased rapidly 3-5 days after DENV-2 S221 challenge, whereas pVD1-CRR vaccinated mice had 30-fold, 40-fold, and at least 1800-fold lower viremia 3, 4, and 5 DPC (all CRR mice $<10 \mathrm{FFU} / \mathrm{mL}$ ) than did WT immunized mice. Virus titers were $\log _{10}$ transformed and the transformed data analyzed by two-way ANOVA $(p<0.0001)$. Bonferroni post hoc test significance is depicted with asterisks, error bars represent SEM. 3, 4, and 5 DPC $n=4,5$, and 6 for WT and 4, 1, and 3 for CRR immunized mice respectively. morbidity including decreased activity, coat ruffling, and weight loss. pVD1-WT vaccinated mice suffered from severely enhanced DENV-2 pathology, including hemorrhagic manifestations producing 95\% mortality ( $p<0.0001$ compared to naïve). Conversely, pVD1-CRR vaccinated mice exhibited reduced DENV-2 disease enhancement and $68 \%$ survival which did not differ from naïve mouse survival $(100 \%, p=0.0769)$ and yet was much greater than WT vaccinated mouse survival $(4.5 \%, p<0.0001$, the Bonferroni multiple comparison adjusted $\alpha=0.017$; Figure 3D). Sick mice began exhibiting external signs of morbidity late on day 3 and reached terminal endpoints 4.5-5.5 DPC for both groups with one WT vaccinated mouse surviving to $8 \mathrm{DPC}$.
To characterize which humoral immune determinates might be responsible for the reduced disease severity and mortality of CRR vaccinated mice we examined sera from 3, 4, and 5 DPC. Four animals in each vaccine treatment were selected $a$ priori for sacrifice 3 DPC, a time point when mice exhibited no outward signs of morbidity. Four and 5 DPC sera were obtained from terminally sick mice via cardiac puncture just prior to euthanasia. Thus, sample sizes were limited on some of these days for pVD1-CRR vaccinated mice since there was limited mortality relative to $\mathrm{pVD} 1-\mathrm{WT}$ vaccinated mice. Serum specimens were collected and DENV-2 $\mathrm{Nt}_{50}$, viremia, and ES-ELISA titers were determined. DENV-2 neutralization was strongly correlated with 
survival; pVD1-CRR immunized mice exhibited a rapid, large magnitude rise in DENV-2 neutralization whereas in pVD1-WT immunized mice, neutralization was lower and increased more slowly ( $p<0.0001$ in a two-way ANOVA, Figure 3E). Three DPC $\mathrm{Nt}_{50}$ titers of $\mathrm{pVD1-CRR}$ vaccinated mice were nearly 100 times greater than those of $\mathrm{pVD} 1-\mathrm{WT}$ vaccinated mice $(\mathrm{CRR}=2783.6$, $\mathrm{WT}=29.5$; Bonferroni post hoc test $p<0.001$ ). This was a $>100$ fold increase in DENV-2 neutralization compared to pre-challenge for CRR vaccinated mice whereas it was a twofold decrease over the same time period for WT vaccinated mice. $\mathrm{Nt}_{50}$ titers of CRR vaccinated mice remained high 4 and 5 DPC whereas those of WT vaccinated mice slowly increased and remained twofold lower than CRR vaccinated mice by 5 DPC. Viremia was negatively correlated with DENV-2 neutralization and positively correlated with mortality (Figure 3F). 3 DPC mean viremia of WT vaccinated mice was 33 times higher $(2325 \mathrm{FFU} / \mathrm{mL})$ than that of CRR vaccinated mice $(70 \mathrm{FFU} / \mathrm{mL}$; Bonferroni post hoc test $p<0.001$ ) with two of four CRR immunized mouse sera below the limits of assay detection (10 FFU/mL). WT viremia continued to increase 4-5 DPC and by 5 DPC was at least 1800 times higher than for CRR immunized mice where all individuals had dropped to below detectable levels (ave $=1.8 \times 10^{4}$ and $<10 \mathrm{FFU} / \mathrm{mL}$ for WT and CRR immunized mice respectively; $p<0.001$ with Bonferroni post hoc test in two-way ANOVA). These findings imply that even though some CRR vaccinated mice were terminally ill 5 DPC, they had cleared their viremia, whereas WT vaccinated mice still exhibited increasing viremia during this time of maximal mortality. Thus, the rapid, large magnitude increase in DENV-2 neutralization of pVD1-CRR vaccinated mice was consistent with an anamnestic response and/or potentially redirected immunity, whereas the slow rise in DENV-2 neutralization of WT vaccinated mice was more characteristic of a primary immune response.

pVD1-WT vaccinated AG129 mice suffering from lethally enhanced DENV-2 disease exhibited pathology consistent with $\mathrm{ADE}$ disease described in passive transfer studies with this mouse model (Balsitis et al., 2010; Zellweger et al., 2010). 3 DPC neither vaccinated nor naïve mice exhibited visible pathological symptoms upon necropsy. All terminally ill mice however had pale, blood-depleted livers, increased intestinal capillary blood flow, and enlarged gaseous stomachs, symptomatic of fluid accumulation caused by vascular leakage. Only WT vaccinated mice exhibited the most severe gastrointestinal hemorrhage (Figure 4A). Although gross pathology upon necropsy of vaccinated mice was not observed until 4 DPC, H\&E staining of liver tissue revealed hepatitis pathology 3 DPC with increasing severity 4 and 5 DPC. Consistent with the reduced gross morbidity and mortality of pVD1-CRR compared to pVD1-WT vaccinated mice, CRR vaccinated mice reaching terminal endpoints exhibited mild lymphoplasmacytic portal, multifocal suppurative, and necrotizing hepatitis, including vacuolar vein congestion. However, pVD1-WT vaccinated mice exhibited moderate to severe lymphoplasmacytic, necrotizing, and multifocal portal hepatitis and in some individuals extensive vascular thrombosis and advanced vacuolar degeneration (Figure 4B). Severe DENV pathology via ADE posits that there should be increased infection of $F \mathrm{C} \gamma \mathrm{R}$ bearing monocytic cells in humans (Morens, 1994) and in AG129 mice,
A

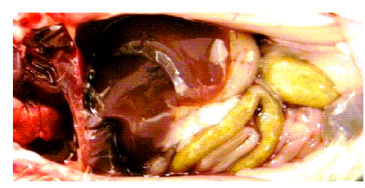

Naïve 3 DPC

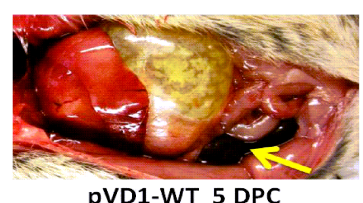

pVD1-WT 5 DPC

B

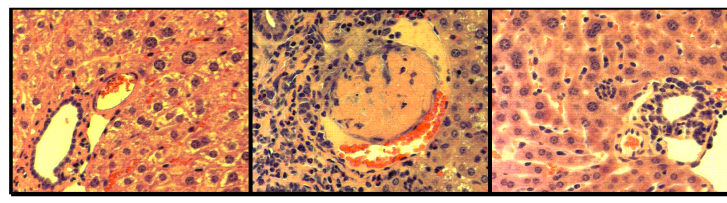

Uninfected

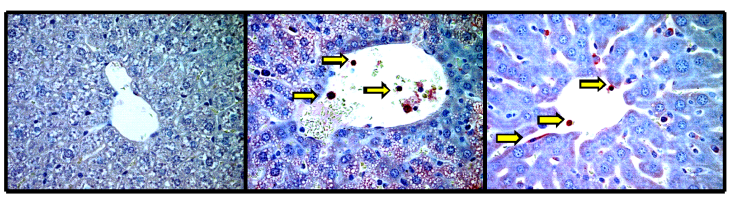

Uninfected

pVD1 -WT

pVD1 -CRR

C

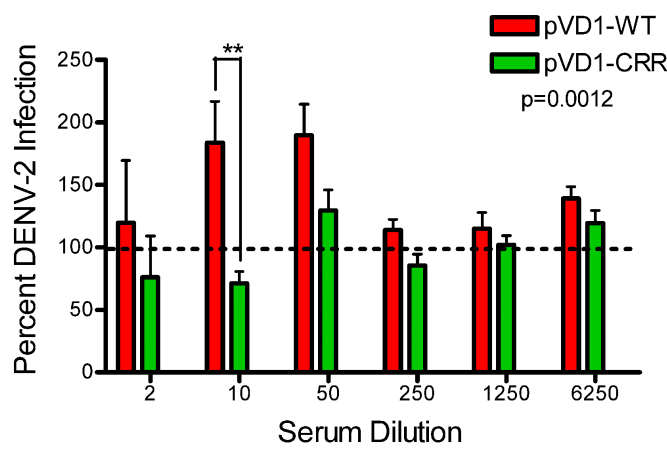

FIGURE 4 | Pathophysiology of enhanced DENV disease in vaccinated AG129 mice. (A) Enhanced DENV disease associated vascular leak pathology of pVD1-WT and -CRR vaccinated mice following sub-lethal heterologous DENV-2 infection. No pathology was visible in naïve or vaccinated mice $3 \mathrm{DPC}$, yet by 4 and 5 DPC mice succumbing to enhanced disease exhibited severe vascular leakage associated pathology. The same mouse is shown in the bottom two photos to highlight the severe intestinal hemorrhage observed in pVD1-WT but not in pVD1-CRR vaccinated mice (arrows). (B) Histology of uninfected, pVD1-WT, and pVD1-CRR vaccinated mouse livers 3 DPC. The top row is hematoxylin and eosin stained liver sections and the bottom row is immunohistochemistry for DENV-2 NS1 protein of the same individual animals. NS1+ mononuclear inflammatory cells and sinusoidal endothelial cells stain red in their cytoplasm and are visible (arrows) in liver tissue from vaccinated mice but not uninfected mice. Multiple sections from multiple animals were examined and single representatives are shown. All photos were taken at 400x magnification. (C) In vitro DENV-2 enhancement by pVD1-WT and pVD1-CRR vaccinated serum 12 weeks following vaccinations and one day prior to DENV-2 challenge. Consistent with the In vivo results, pVD1-WT vaccinated serum significantly enhanced DENV-2 infection whereas pVD1-CRR vaccinated serum did not $(p=0.0012)$. The data are representative of two independent experiments of 4 pools of 6-7 individual serum specimens for each vaccine treatment and were analyzed with two-way ANOVA and Bonferroni post hoc test significance at individual dilutions is depicted with asterisks. 
previous studies found that antibody-enhanced mortality was associated with increased DENV infection and replication in liver sinusoidal endothelial cells (Balsitis et al., 2010; Zellweger et al., 2010). To assess viral replication in liver tissue we used immunostaining for DENV-2 NS1 protein which is expressed during viral replication. Of note, although we did not attempt to quantify differences between WT and CRR vaccine treatments, mononuclear inflammatory cells in the portal areas and sinusoidal endothelial cells of both WT- and CRR vaccinated liver tissue were NS1 positive by $3 \mathrm{DPC}$, suggestive of active viral replication in $\mathrm{Fc} \gamma \mathrm{R}$ bearing cells of the liver, and liver tissue of naïve mice was negative for NS1 by immunostaining (Figure 4B). Lastly, pre-challenge pVD1-WT vaccinated mouse serum (1 day prior to DENV-2 challenge) significantly enhanced DENV-2 replication in Fc $\gamma \mathrm{R}$ bearing human K562 cells, and pVD1-CRR immunized mouse sera did not (Figure 4C). In summary, the high levels of DENV-1 vaccine induced cross-reactive $\mathrm{EDII}_{\mathrm{FP}}$ IgG prior to challenge, increased pathology and mortality, higher viremia, and active viral replication in Fc $\gamma \mathrm{R}$ bearing cells of WT immunized mice are all consistent with previous descriptions of antibody-enhanced DENV disease in AG129 mice (Balsitis et al., 2010; Zellweger et al., 2010).

\section{Cross-reactivity reduced DNA vaccination sculpts immune memory so that response to virus infection is redirected: reducing pathological and increasing protective immunity}

We used DENV-1 and DENV-2 ES-ELISA to quantify and differentiate humoral immune responses to DENV-1 vaccination from DENV-2 challenge. Supporting the hypothesis that WT vaccinated mice were slower to mount DENV-2 specific immunity due to DENV-1 primed cross-reactive memory responses, WT vaccinated mice had lower DENV-2 total IgG titers yet larger populations of both DENV-1 and DENV-2 EDII ${ }_{\mathrm{FP}}$ IgG than did CRR vaccinated mice following heterologous challenge (Figure 5). Total DENV-1 IgG titers did not differ between vaccine treatments 3, 4, and 5 DPC with DENV-2 (Figure 5A); however, DENV-2 IgG titers were higher for $\mathrm{pVD} 1-\mathrm{CRR}$ than for $\mathrm{pVD} 1-\mathrm{WT}$ immunized mice (Figure 5B). Nevertheless, pVD1-WT vaccinated mice had significantly larger populations of $\mathrm{EDII}_{\mathrm{FP}} \mathrm{IgG}$ for both DENV-1 (Figure 5C) and DENV-2 (Figure 5D) than did pVD1CRR vaccinated mice. Not only did pVD1-WT mice have larger

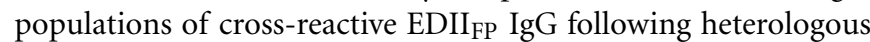
challenge, there was also a significant increase in DENV-1 EDII ${ }_{F P}$ IgG post challenge when compared to the 12 week pre-challenge data (Figure 5C). DENV-1 EDII $\mathrm{FP}$ IgG GMT of WT immunized mice increased from $17.7 \pm 4.6 \%$ pre-challenge to $35.5 \pm 5.4 \%$ of the total IgG response post DENV-2 challenge. However, there

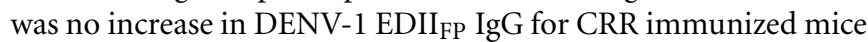
before and after challenge ( $p=0.364$, Mann-Whitney $U)$. We also analyzed individual pairs of pre- and post-DENV-2 challenge

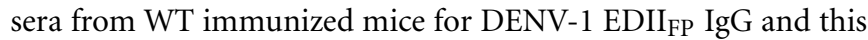
analysis also demonstrated a significant increase post-challenge $(p=0.0138)$ and a significant effect of individual serum pairing ( $p=0.0267$, one-tailed paired $t$-test; data not shown). Together, these results suggest a strong memory response to cross-reactive $\mathrm{E}$ protein epitopes in DENV-2 that were primed by pVD1-WT vaccination and that such an immunodominant anamnestic response was lacking in pVD1-CRR immunized mice.
ES-ELISA can also determine the proportion of IgG recognizing $\mathrm{E}$ epitopes outside of the manipulated $\mathrm{EDII}_{\mathrm{FP}}$ and $\mathrm{EDIII}_{\mathrm{CR}}$ antigenic regions in the $\mathrm{pVD1}-\mathrm{CRR}$ vaccine (non-EDII ${ }_{\mathrm{FP}} \mathrm{EDIII}_{\mathrm{CR}}$ IgG; Figure 1;Hughes et al., 2012a). We hypothesized that pVD1CRR immunized mice, with their reduced EDII ${ }_{F P}$ response, should exhibit increased capacity to redirect humoral immune responses to increase the DENV-2 non-EDII ${ }_{\mathrm{FP}} \mathrm{EDIII}_{\mathrm{CR}}$ antibody populations concomitant with their reduced EDII $_{\mathrm{FP}}$ response and that WT immunized mice should show the opposite pattern. Supporting this prediction, CRR vaccinated mice had significantly larger populations of DENV-2 non-EDII ${ }_{\mathrm{FP}} \mathrm{EDIII}_{\mathrm{CR}} \mathrm{IgG}_{3}$, 4, and $5 \mathrm{DPC}$ with DENV-2 than did pVD1-WT immunized mice $(p=0.0025$ in two-way ANOVA; Figure 5E). This was the same time period that WT immunized mice exhibited maximum enhanced DENV-2 disease mortality (Figure 3D) and significant increases in EDII ${ }_{F P}$ IgG (Figures 5C,D); whereas CRR immunized mice exhibited 100fold increased DENV-2 neutralization (Figure 3E). Moreover, a comparison of DENV-1 non-EDII ${ }_{\mathrm{FP}}$ EDIII $_{\mathrm{CR}}$ IgG for individual WT immunized mice pre- and post-challenge showed a significant decrease $(p=0.0435)$ in this antibody population 3-5 DPC and a significant effect of individual mouse serum pairing on this decrease ( $p=0.0111$, one-tailed paired $t$-test; data not shown). A decrease in this DENV-1 antibody population of WT immunized mice is what one would expect if there is a trade-off between immunodominant secondary responses to $\mathrm{EDII}_{\mathrm{FP}}$ and non-EDII ${ }_{\mathrm{FP}} \mathrm{EDIII}_{\mathrm{CR}}$ epitopes. Antibody stimulated from epitopes outside $\mathrm{EDII}_{\mathrm{FP}}$ and $\mathrm{EDIII}_{\mathrm{CR}}$ antigenic regions altered in our pVD1-CRR vaccine include neutralizing DENV complex reactive antibodies such as MAb 1 B7 primed by either vaccine and boosted by DENV-2 challenge (Table 2 ) and/or DENV-2 serotypespecific neutralizing antibodies stimulated only by the challenge virus.

Three non-mutually exclusive mechanisms could explain the rapid increase in DENV-2 neutralizing antibody by CRR immunized mice 3 DPC: (1) anamnestically increased complex crossreactive neutralizing antibody not altered by the substitutions introduced in the pVD1-CRR vaccine (e.g., 1B7-like), (2) primarily increased DENV-2-specific neutralizing antibody, or (3) increased relative neutralizing capability of CRR vaccinated sera by these or other neutralizing antibody populations due to a lack of steric interference by the large populations of EDII $\mathrm{FP}_{\mathrm{FG}}$ present in WT vaccinated mice (Ndifon et al., 2009). To test if the increased $\mathrm{Nt}_{50}$ titers of CRR vaccinated mice were due to increases in DENV complex cross-reactive neutralizing antibodies we determined the $\mathrm{Nt}_{50}$ titers for CRR and WT vaccinated mouse serum $3 \mathrm{DPC}$ against DENV-1, DENV-3, and DENV-4 (Figure 5F). The mean $\left(n=4\right.$ each) $\mathrm{Nt}_{50}$ titers for CRR and WT vaccinated mice respectively were 46 and 33 for DENV-1 $(p=0.066), 55$ and 27 for DENV-3 ( $p=0.0009)$, and 147 and 42 for DENV-4 $(p=0.0178$; one-tailed $t$-test for all). These 1.5-, 2.0-, and 3.5-fold higher DENV-1, -3 , and $-4 \mathrm{Nt}_{50}$ titers for CRR vaccinated mice did not approach the nearly 100 -fold greater DENV-2 $\mathrm{Nt}_{50}$ titer of CRR immunized mice 3 DPC (2785 and 29 respectively). These data suggest that CRR vaccinated mice appear to have increased complex cross-reactive neutralizing antibody relative to WT vaccinated mice; but this antibody class either neutralizes DENV-2 more efficiently than other DENV serotypes, or there are also increased 


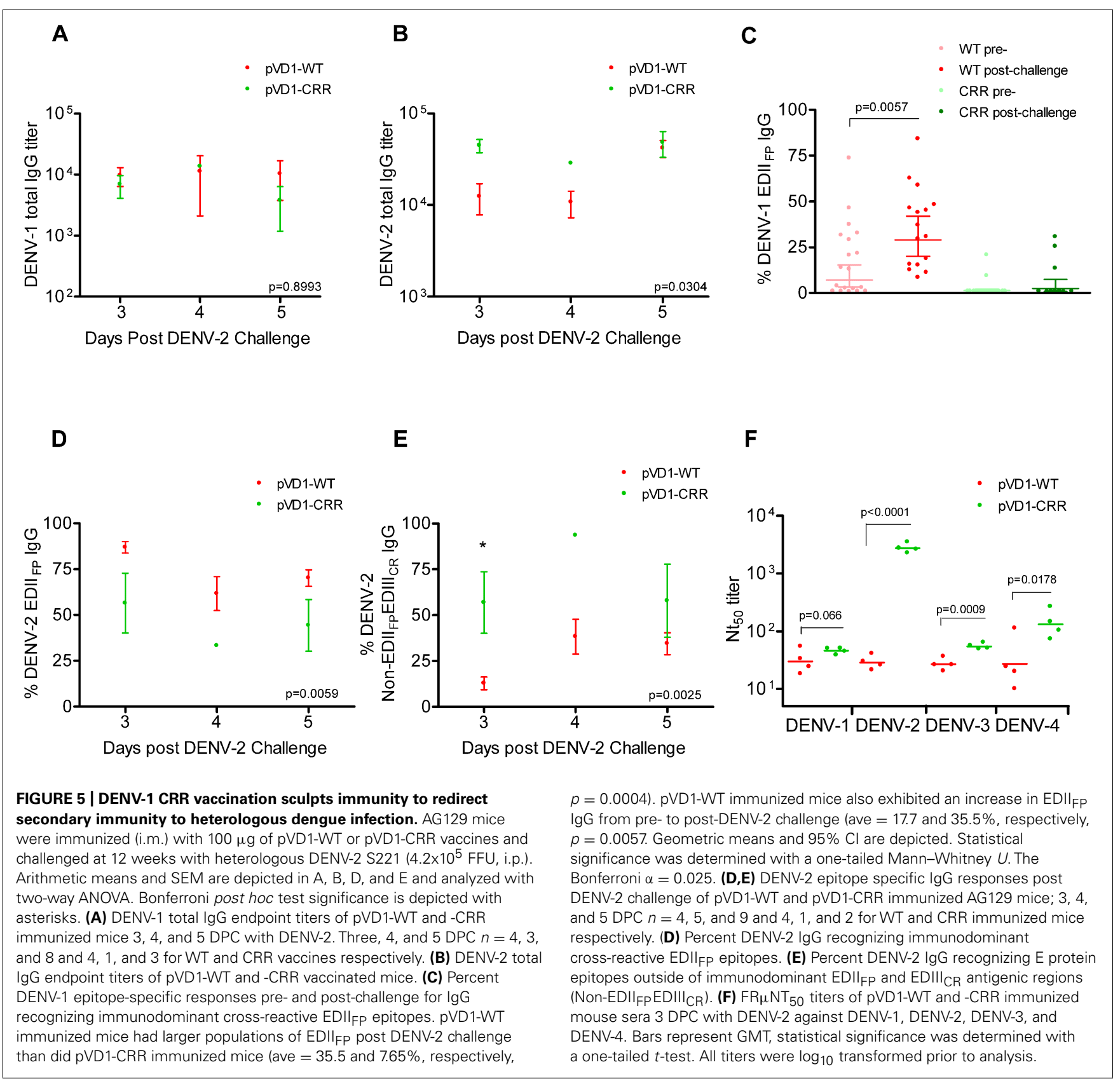

proportions of DENV-2 specific neutralizing antibody in CRR vaccinated serum by 3 DPC with DENV-2.

To further characterize the epitopes recognized by the antibodies responsible for the rapid rise in DENV-2 neutralization observed in CRR vaccinated mice, we tested the ability of 3 DPC sera to block the binding of labeled MAbs in a DENV2 epitope-blocking ELISA. We examined the blocking by WT and CRR vaccinated mouse sera for MAbs recognizing complex cross-reactive epitopes present in both DENV-1 vaccines and in the DENV-2 challenge virus (1B7, 10-D35A, and D35C9-1), MAbs recognizing sub-complex cross-reactive epitopes knocked out in the CRR vaccine but present in the WT vaccine and DENV-2 challenge virus (1A1D-2 and 9D12), and MAbs

recognizing DENV-2 serotype-specific epitopes present only in the challenge virus (9A3D-8 and 3H5). Surprisingly, pVD1-CRR vaccinated mouse sera exhibited significantly greater blocking than did WT vaccinated sera for all of these MAbs with the exception of D3-5C9-1 the only non-neutralizing MAb against DENV-2, implying that CRR immunized mice had higher titers of antibody recognizing the same or similar epitopes as these labeled MAbs (Figure 6). The greatest relative increase in blocking was for DENV complex, neutralizing MAb 1B7 which exhibited 16-fold greater blocking by CRR than the $2.25 \%$ blocking by WT ( $p=0.0072$, two-tailed $t$-test), implying greater 1B7like neutralizing antibody in CRR vaccinated mouse sera than in WT mouse sera, and consistent with increased anamnestic 


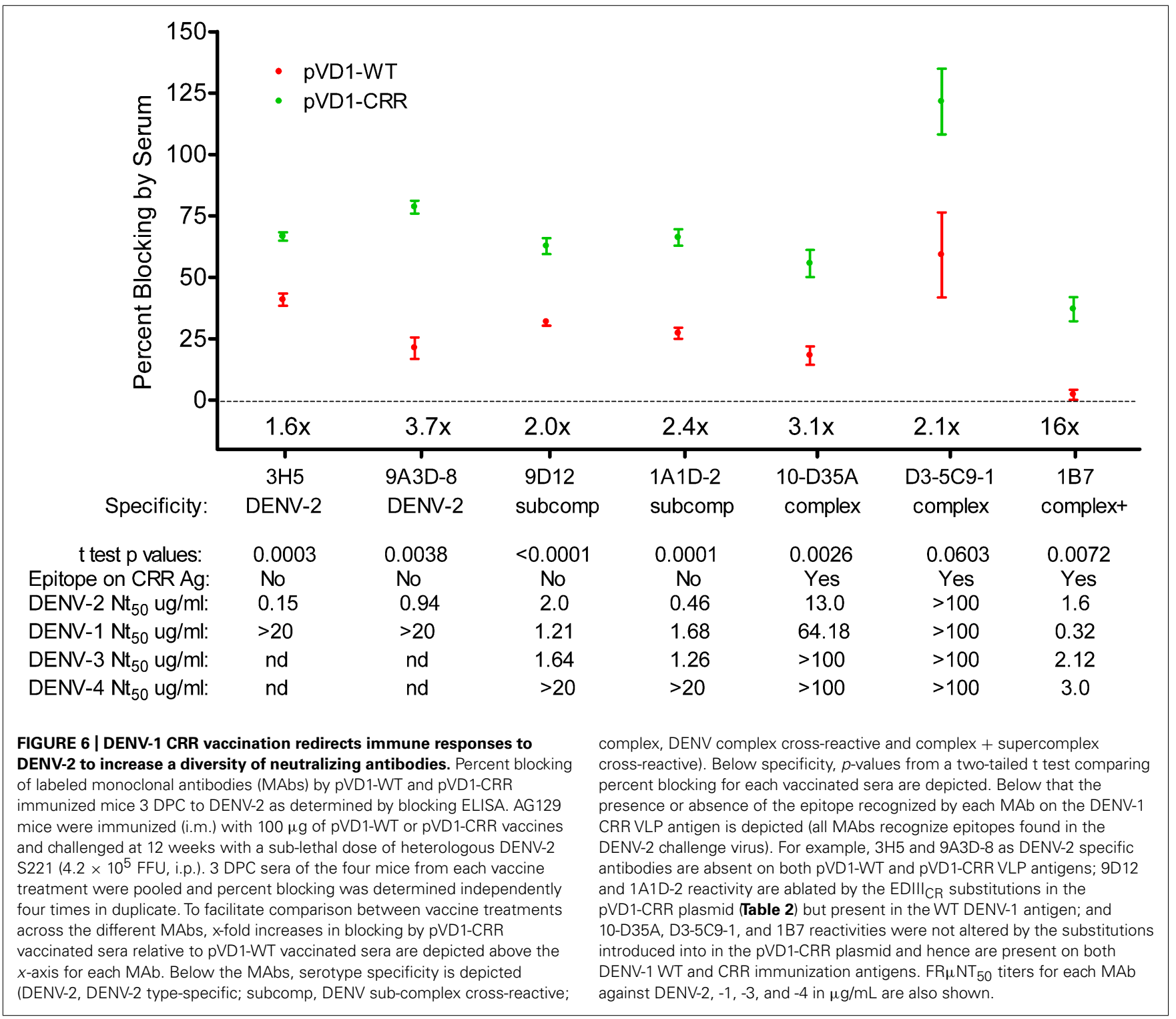

induction of normally sub-dominant 1B7-like antibody in CRR immunized mice. CRR vaccinated mice also had 3.7 and 1.6-fold larger populations of DENV-2 serotype-specific potently neutralizing $3 \mathrm{H} 5$ and $9 \mathrm{~A} 3 \mathrm{D}-8$ like antibodies than did WT vaccinated mice ( $p=0.0003$ and $p=0.0058$, respectively), $2.4-$ and 2fold greater sub-complex cross-reactive neutralizing $1 \mathrm{~A} 1 \mathrm{D}-2$ and 9D12 like antibodies ( $p=0.0001$ and $p<0.0001$ respectively), and 3.1-fold more DENV complex cross-reactive weakly neutralizing $10-\mathrm{D} 35 \mathrm{~A}$ like antibodies $(p=0.0026)$. There was a non-significant 2.1-fold increase in complex cross-reactive MAb D3-5C9-1. We next determined the neutralizing capabilities of these seven MAbs since not all had been previously published in the literature (Figure 6). Not surprisingly, DENV-2 serotype-specific MAbs 3H5 and 9A3D-8 exhibited potent DENV-2 neutralization $\left(\mathrm{Nt}_{50}=0.15\right.$ and $0.94 \mu \mathrm{g} / \mathrm{mL}$ respectively). 1A1D-2 also potently neutralized DENV-2 $(0.46 \mu \mathrm{g} / \mathrm{mL})$ although it neutralized DENV-1 and DENV-3 about threefold less than DENV-2, similar to the DENV-2 neutralizing capabilities of both 9D12 and 1B7. 10-D35A exhibited weak neutralization of DENV-2 $(13.04 \mu \mathrm{g} / \mathrm{mL})$, and D3-5C9-1 did not neutralize any DENV serotype. Together, these results begin to tease apart the individual humoral immune components responsible for the polyclonal response of vaccinated mice to heterologous infection. The 100fold increase in DENV-2 neutralization appeared to be due to a combination of large increases in cross-reactive neutralizing antibodies, similar to $1 \mathrm{~B} 7$, but also to relative increases and large populations of potently neutralizing serotype-specific and sub-complex cross-reactive antibodies. Thus, in response to heterologous DENV-2 infection, pVD1-CRR immunized mice exhibited rapid increases in neutralizing antibody populations with diverse patterns of reactivity that not only increased protection from enhanced DENV-2 disease mortality but also exhibited increased neutralization breadth across the DENV complex. 


\section{DISCUSSION}

In this report we constructed WT and CRR DENV-1 DNA vaccine candidates and compare their humoral immunogenicity as it relates to vaccine safety and efficacy using the AG129 mouse dengue disease model. The CRR vaccine was specifically engineered as a second generation vaccine candidate to reduce the potential for vaccine-induced susceptibility to severe dengue disease via ADE, a theoretical safety concern for dengue vaccine development (Miller, 2010; Murphy and Whitehead, 2011; Thomas, 2011; Heinz and Stiasny, 2012). CRR immunized mice had significantly reduced titers of $\mathrm{EDII}_{\mathrm{FP}}$ antibodies, induced from cross-reactive immunodominant epitopes associated with $\mathrm{ADE}$ and increased pathology in humans and dengue animal models (Goncalvez et al., 2007; Balsitis et al., 2010; Beltramello et al., 2010; Dejnirattisai et al., 2010; Zellweger et al., 2010). We further demonstrate that by disrupting these epitopes we can redirect the immunodominance hierarchy away from pathogenic toward normally subdominant, potently neutralizing epitopes. WT vaccinated mice had high EDII $_{\mathrm{FP}}$ antibody titers and enhanced a heterologous, sub-lethal DENV-2 infection into a DHF-like disease pathology resulting in $95 \%$ mortality. CRR vaccinated mice however, lacking measurable $\mathrm{EDII}_{\mathrm{FP}}$ antibodies, exhibited increased protective, neutralizing immunoglobulins, and had significantly reduced morbidity and mortality that did not differ from naive mice.

The location of E protein substitutions introduced into the pVD1-CRR vaccine construct used in this study was based upon previously published work in DENV-2 (Crill et al., 2009; Hughes et al., 2012a). We previously constructed DENV-2 CRR DNA vaccines with substitutions knocking out cross-reactive epitopes in the EDII ${ }_{F P}$, in EDIII, and in both EDII FP and EDIII and compared their immunogenicity with each other and with the WT DENV-2 vaccine (Hughes et al., 2012a). All vaccines induced similar high-titer DENV-2 neutralizing antibody responses in Swiss Webster mice. In in vitro ADE assays, sera from WT immunized mice significantly enhanced DENV-1, -2 , and -3 replication and sera from mice immunized with $\mathrm{EDII}_{\mathrm{FP}}$ or EDIII substitutions enhanced DENV-1 or DENV-3, only serum from mice immunized with vaccines containing CRR substitutions in both EDII $\mathrm{FP}$ and EDIII lacked enhancing capabilities for all serotypes at the lowest tested serum dilution (1:2). We observed that vaccines containing substitutions in EDIII exhibited a non-significant trend toward reduced DENV-2 neutralizing antibody titers but that the vaccine containing substitutions in both EDII $_{\mathrm{FP}}$ and EDIII also showed the greatest increase in heterologous neutralization. Based upon these observations we decided to incorporate substitutions in both EDII $_{\mathrm{FP}}$ and EDIII into the DENV-1 CRR vaccine construct.

Our results, suggestive of vaccine-induced severe dengue disease and mortality via ADE, are consistent with and supported by two recent studies of ADE-induced dengue disease in AG129 mice using passive transfer of DENV immune sera (Balsitis et al., 2010; Zellweger et al., 2010). These studies found that administering $10^{4}, 10^{5}$, or $10^{6}$ pfu of DENV-2 S221 was sub-lethal to naïve AG129 mice receiving passively transferred normal mouse serum $24 \mathrm{~h}$ prior to challenge. However, mice receiving heterologous DENV-1, -3 , or -4 immune sera $24 \mathrm{hr}$ prior to challenge died
4-5 DPC from severe dengue disease resembling DHF. The disease pathology was similar to that seen in human DHF. Confirmation that this enhanced DHF-like disease and mortality resulted from ADE was supported by passive transfer of enhancing EDII ${ }_{F P}$ MAb $4 \mathrm{G} 2$ and by rescue from enhanced mortality by passive transfer of 4G2 F(ab')2 fragment unable to bind Fc $\gamma \mathrm{R}$ (Balsitis et al., 2010). In this study, we found that when exposed to normally sub-lethal doses of DENV-2 S221, pVD1-WT vaccinated mice suffered 95\% mortality, shared many of these same symptoms and that the high viremia and enhanced disease pathology and mortality were significantly reduced by immunization with a modified pVD1-CRR vaccine.

An important finding in this study was the rapid induction of diverse DENV-2 neutralizing antibodies by pVD1-CRR vaccinated mice in response to heterologous DENV-2 infection. There was a large increase in 1B7-like antibody for CRR vaccinated mice, supporting the importance of this class of neutralizing antibody. However, the majority of the increase in DENV-2 neutralization by CRR vaccinated mice appeared to be due to DENV-2 serotype-specific neutralizing antibody, since DENV-1, -3 , and -4 neutralization only increased moderately. Neither pVD1-WT nor pVD1-CRR immunized mice were primed for DENV-2 serotypespecific neutralizing antibody responses, how then could this antibody class be so potently increased during early acute heterologous DENV-2 infection in CRR immunized mice? A recent study examining humoral and cellular immune response to primary DENV-1 and secondary DENV-2 infections in AG129 mice is relevant to this discussion (Zompi et al., 2012). These authors observed that by 6 days post-infection naïve B cells had differentiated into plasma cells capable of producing both serotype-specific and cross-reactive neutralizing antibody. Notably though, DENV2-specific plasma cells peaked at 6 days post-primary DENV-2 infection, but in secondary DENV-2 infected mice this peak was delayed until 9 days post-infection. These findings were interpreted to suggest that secondarily infecting DENV-2 antigen was more readily captured by cross-reactive DENV-1 derived memory $\mathrm{B}$ cells and/or antibody, preventing the early binding and activation of naïve B cells during secondary DENV-2 infection. A similar phenomenon could explain the more rapid production of DENV-2 serotype-specific neutralizing antibody in pVD1-CRR immunized mice by 3 DPC with DENV-2. We believe such a process to be a major mechanistic explanation behind the immune redirection associated with the reduced DENV-2 disease enhancement and mortality observed in pVD1-CRR immunized mice.

A surprising finding was the rapid increase of sub-complex cross-reactive antibodies in pVD1-CRR immunized mice following DENV-2 infection, since antibodies recognizing epitopes similar to MAbs 1A1D-2 and 9D12 should have been primed by pVD1-WT but not by pVD1-CRR vaccination. One possible explanation is that the CRR substitutions introduced into EDIII did not completely knock-out these epitopes. This is possible because EDIII $_{\mathrm{CR}}$ antibodies recognize epitopes in a complex, conformational antigenic surface (Sukupolvi-Petty et al., 2007; Gromowski et al., 2008; Pierson et al., 2008; Cockburn et al., 2012). However, the increased production of sub-complex cross-reactive antibodies in CRR vs. WT vaccinated mice may simply be due to a reduced capability of WT vaccinated mice to produce this sub-dominant 
antibody class in response to DENV-2 infection (Zompi et al., 2012). This study did not address the possible role of antibody interference in explaining the rapid increases in DENV-2 neutralization observed in $\mathrm{PVD1}$-CRR vaccinated mice (Ndifon et al., 2009). Weakly or non-neutralizing antibodies recognizing EDII $\mathrm{FP}_{\mathrm{PP}}$ epitopes could sterically interfere with the binding of potently neutralizing antibodies recognizing either EDIII or recently identified inter-dimer quaternary epitopes (de Alwis et al., 2012). The lack of large populations of EDII $\mathrm{FP}$ IgG in pVD1-CRR vaccinated mouse serum can therefore increase its relative neutralization and we suspect this phenomenon to also play a role in the increased neutralization of pVD1-CRR vaccinated mice.

Because this study utilized active vaccination, the increased survival and decreased disease incidence and severity of pVD1CRR vaccinated mice could result from additional factors such as differences in cellular immunity. Even in IFN $\alpha / \beta$, IFN $\gamma$ receptordeficient AG129 mice, cellular immunity could play an important role in either protection from or enhancement of dengue infection and disease (Yauch et al., 2009, 2010; Zompi et al., 2012). As a part of a recently published $\mathrm{CD} 4+\mathrm{T}$ cell study we developed an overlapping peptide library for DENV-2 prM and E proteins. Mice immunized with DENV-2 CRR vaccines, containing substitutions at the same EDII ${ }_{\mathrm{FP}}$ and EDIII $_{\mathrm{CR}}$ residues, did not differ in CD4+ or CD8 + T cell recognition for the WT DENV-2 peptides, implying that the substitutions introduced into DENV CRR vaccines are either not located in $\mathrm{T}$ cell epitopes or do not alter existing $\mathrm{T}$ cell epitopes in these regions (Hughes et al., 2012b; Hughes and Chang, unpublished data).

Most immunodominant DENV T cell epitopes have been localized to the non-structural proteins, particularly NS3, and the DNA vaccines utilized in this study do not contain the genes coding for non-structural proteins (Mathew and Rothman, 2008). T cell epitopes that have been localized to the $\mathrm{prM} / \mathrm{E}$ structural proteins have predominately been localized to regions outside those modified in our CRR vaccine (Yauch et al., 2009; Duangchinda et al., 2010). Roehrig et al. (1994) found that a DENV-2 E protein

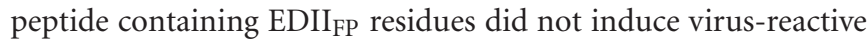
$\mathrm{CD} 4+\mathrm{T}$ cells in vitro or prime $\mathrm{H}-2 \mathrm{~b} \mathrm{CD} 4+$ virus-specific $\mathrm{T}$ cells whereas peptides spanning EDIII residues $302-333$ or $352-368$ could weakly induce such priming. It is therefore possible that pVD1-CRR substitutions could increase CD4+ T cell priming beyond the low levels observed with the WT peptides to boost humoral immunity or that the EDII $I_{\mathrm{FP}}$ and/or EDIII $\mathrm{CR}_{\mathrm{CR}}$ substitutions could increase $\mathrm{CD} 8+\mathrm{T}$ cell activation relative to $\mathrm{WT}$ vaccine; possibly accounting for the observed survival differences. However, similar pathologies were observed following passive transfer of DENV immune sera (Balsitis et al., 2010; Zellweger et al., 2010). In the present study, we observed in vitro DENV-2 enhancement with pVD1-WT vaccinated mouse sera that was not observed with pVD1-CRR vaccinated sera; and in our recently published study, enhanced disease mortality of AG129 mice was similarly reduced following passive transfer of DENV-2 CRR vaccinated mouse sera (Hughes et al., 2012a). Together, these findings support the interpretation that the enhanced DHF-like disease and mortality of WT immunized mice and its significant reduction in $\mathrm{pVD} 1-\mathrm{CRR}$ vaccinated mice, was most likely due to the observed alterations of humoral immunity.
The pVD1-CRR vaccine exhibited an improved safety profile compared to WT in an in vivo AG129 model, however, some CRR vaccinated mice still succumbed to DENV disease, implying either that there could be additional pathogenic antibody classes not targeted by our CRR modifications or that mechanisms in addition to ADE may have been responsible for the mortality observed in pVD1-CRR vaccinated mice. Cross-reactive, non-neutralizing antibody recognizing prM has recently been reported to comprise a significant portion of the human anti-DENV response and such antibody can enhance DENV replication (Beltramello et al., 2010; Dejnirattisai et al., 2010; Rodenhuis-Zybert et al., 2010). However, the limited data suggest that prM antibodies may not be as frequently induced in mice as in humans (Henchal et al., 1985; Roehrig et al., 1998); this could be the result of different routes of infection, needle (i.m.) versus mosquito (i.d.) or of the different forms of virus (mature, immature, or partially mature) being delivered. Nevertheless, VLPs produced by our flavivirus and DENV plasmids in tissue culture do contain prM and vaccination with these plasmids can produce antibody recognizing prM (Chang et al., 2003; Chiou et al., 2012). CRR substitutions introduced into these plasmids do not alter the prM processing or change the relative ratios of VLP prM and $\mathrm{E}$ or their induced antibody populations in comparison to WT (Chiou et al., 2012). Thus, prM antibodies may be of concern in human vaccination and they could explain the residual enhancement observed in CRR vaccinated AG129 mice (Henchal et al., 1985; Roehrig et al., 1998), but they are unlikely to account for the differences in pathology and mortality between pVD1-CRR and -WT immunized mice in this study. Alternatively, residual CRR vaccinated mouse enhancement could have occurred via antibody recognizing E protein epitopes outside of the CRR modifications and/or from lower, non-protective levels of normally protective antibodies (Pierson et al., 2008; Beltramello et al., 2010; Hughes et al., 2012a).

Antibody-mediated neutralization and enhancement are two phenomena that exist along a continuum of antibody concentration. Antibody-mediated neutralization of flaviviruses requires virion loading with a stoichiometry that exceeds the neutralization threshold. This neutralization threshold varies for different antibodies based upon their affinity and epitope accessibility. When antibody occupancy does not exceed this neutralization threshold, enhancement can occur (Pierson et al., 2008). EDII FP epitopes are relatively inaccessible on mature and partially mature virions and antibodies recognizing these epitopes typically require high to saturated occupancy if they are to neutralize virus at all. Such antibodies therefore have the potential to enhance infection across a wide range of concentrations. Serotype-specific potently neutralizing antibodies recognizing EDIII lateral ridge epitopes such as DENV-2 specific 3H5 and 9A3D-8 however, are able to neutralize virus at much lower occupancy thresholds (Pierson et al., 2008). The implication of these findings is that antibody recognizing EDII $_{\mathrm{FP}}$ epitopes will tend to enhance infection at most concentrations and only neutralize at very high concentrations whereas EDIII serotype-specific antibodies will neutralize across most concentrations and only enhance at the lowest concentrations. Thus, the reduced levels of cross-reactive EDII $\mathrm{FP}$ recognizing antibody and increased levels of DENV-2 specific antibody in pVD1-CRR 
immunized mice supports their playing important roles in the observed differences in morbidity and mortality.

The concern of vaccine-induced immunodominant antibody responses in dengue vaccinology and our approach of genetically modifying these pathogenic epitopes to redirect the immunodominance hierarchy have parallels with other multi-strain pathogens such as HIV and Influenza. Both HIV and influenza vaccinology have to address original antigenic sin and strain-specific immunodominance. For all of these viruses, producing efficacious and improved next-generation vaccines is likely to require altering the native, WT immune responses (Ndifon et al., 2009; Miller, 2010; Durbin and Whitehead, 2011; Nara et al., 2011; Schmitz et al., 2011). In both HIV and influenza there has been interest in this area of modifying immunogens to redirect immune responses, typically referred to as immune dampening and immune refocusing. Some generalizations from this body of work are consistent with and support our CRR DENV DNA vaccination results. The dampening of immunodominant epitopes resulted in decreased induction of antibodies recognizing the targeted epitopes while increasing the amount of antibody stimulated from natively subdominant epitopes. Moreover, in spite of such major alterations in immunodominance hierarchies, antigenically modified immunogens induced similar total overall antibody titers as did WT immunogens (Tobin et al., 2008).

There is substantial interest in utilizing heterologous vaccine prime-boost strategies to improve and broaden immunogenicity, especially in the context of DNA vaccination (Dale et al., 2006; Chen et al., 2007; Simmons et al., 2010; Ding et al., 2011; Guenaga et al., 2011). In this context, "heterologous" typically refers to the use of different vaccine formats to present the same viral immunogens between prime and boost, most commonly DNA prime and protein (recombinant, inactivated virus, or live attenuated virus) boost. Much of this interest has also been directed toward HIV (Walker and Burton, 2010) and influenza (Wei et al., 2010; Ding et al., 2011) where the vaccine goal is to increase the breadth of neutralization and hence protection from these highly variable multi-strain pathogens. Because of the similarities to DENV and the difficulties of rapidly inducing balanced tetravalent immunity with current DENV live-attenuated vaccines (Guy et al., 2009; Murphy and Whitehead, 2011), DNA prime-protein boost strategies are particularly appealing to DENV vaccinology. We found

\section{REFERENCES}

Balsitis, S. J., Williams, K. L., Lachica, R., Flores, D., Kyle, J. L., Mehlhop, E., et al. (2010). Lethal antibody enhancement of dengue disease in mice is prevented by $\mathrm{fc}$ modification. PLoS Pathog. 6, e1000790. doi: 10.1371/journal.ppat.1000790

Beltramello, M., Williams, K. L., Simmons, C. P., Macagno, A., Simonelli, L., Quyen, N. T., et al. (2010). The human immune response to dengue virus is dominated by highly crossreactive antibodies endowed with neutralizing and enhancing activity. Cell Host Microbe 8, 271-283.

Brehm, M. A., Pinto, A. K., Daniels, K. A., Schneck, J. P., Welsh, R.
M., and Selin, L. K. (2002). T cell immunodominance and maintenance of memory regulated by unexpectedly cross-reactive pathogens. Nat. Immunol. 3, 627-634.

Chang, G. J., Davis, B. S., Hunt, A. R., Holmes, D. A., and Kuno, G. (2001). Flavivirus DNA vaccines: current status and potential. Ann. N. Y. Acad. Sci. 951, 272-285.

Chang, G. J., Davis, B. S., Stringfield, C., and Lutz, C. (2007). Prospective immunization of the endangered California condors (Gymnogyps californianus) protects this species from lethal West Nile virus infection. Vaccine 25, 2325-2330.

that DENV-1 CRR DNA vaccination redirected subsequent immunity in response to DENV-2 challenge and increased the induction of a broad repertoire of neutralizing antibodies to produce a polyclonal DENV-2 neutralizing response with increased crossneutralization to other DENV serotypes. Our findings suggest that CRR DNA vaccines hold potential for novel DENV vaccine strategies that take advantage of the benefits of both DNA and live attenuated virus vaccines. One such strategy is to prime hosts with a low-dose tetravalent CRR DNA vaccine to elicit a beneficial memory response with reduced enhancing capability and limited neutralizing antibody to each serotype. Such priming might allow for efficient tetravalent live-attenuated virus boost 1-2 months later to produce balanced and protective tetravalent immunity. We are currently initiating preclinical studies using this strategy with the goal to achieve protective tetravalent immunity within 3 months; a long sought after goal of DENV vaccinology that continues to elude existing DENV vaccine candidates.

\section{ACKNOWLEDGMENTS}

We thank Danielle Mayer, Michelle Conetta, Shirstine Gordon, and Danielle Holligan for technical assistance and Dr. Sujan Shresta and her lab for the generous gift of DENV-2 S221 virus. Barbara Powers of the Colorado State University Veterinary Teaching Hospital provided histology and pathology interpretations along with Dr. Nord Zeidner (CDC, DVBD) who generously assisted with histology photos and additional interpretations. CDC DVBD is a federally funded national public health lab (USCDC) and all funding for this work was supported by such federal funding. Holly R. Hughes, Nicole B. Trainor, and Matt T. Whitney were supported by a federally funded contract to Columbus Technologies and Services, Inc.

\section{AUTHORS CONTRIBUTION}

Wayne D. Crill, Gwong-Jen J. Chang, and Holly R. Hughes conceived and designed the experiments. Wayne D. Crill, Brent S. Davis, Holly R. Hughes, Nicole B. Trainor, and Matt T. Whitney performed the experiments. Wayne D. Crill, Gwong-Jen J. Chang, and Holly R. Hughes analyzed the data. Wayne D. Crill, Gwong-Jen J. Chang, and Holly R. Hughes contributed reagents and/or materials. Gwong-Jen J. Chang and Holly R. Hughes critically reviewed the paper, and Wayne D. Crill wrote the paper.

Chang, G. J., Hunt, A. R., Holmes, D. A., Springfield, T., Chiueh, T. S., Roehrig, J. T., et al. (2003). Enhancing biosynthesis and secretion of premembrane and envelope proteins by the chimeric plasmid of dengue virus type 2 and Japanese encephalitis virus. Virology 306, 170-180.

Chen, L., Ewing, D., Subramanian, H., Block, K., Rayner, J., Alterson, K. D., et al. (2007). A heterologous DNA prime-Venezuelan equine encephalitis virus replicon particle boost dengue vaccine regimen affords complete protection from virus challenge in cynomolgus macaques. J. Virol. 81, 11634-11639.
Chiou, S. S., Fan, Y. C., Crill, W. D., Chang, R. Y., and Chang, G. J. (2012). Mutation analysis of the cross-reactive epitopes of Japanese encephalitis virus envelope glycoprotein. J. Gen. Virol. 93, 1185-1192.

Cockburn, J. J., Navarro Sanchez, M. E., Fretes, N., Urvoas, A., Staropoli, I., Kikuti, C. M., etal. (2012). Mechanism of dengue virus broad crossneutralization by a monoclonal antibody. Structure 8, 303-314.

Crill, W. D., and Chang, G. J. (2004). Localization and characterization of flavivirus envelope glycoprotein cross-reactive epitopes. J. Virol. 78, 13975-13986. 
Crill, W. D., Hughes, H. R., Delorey, M. J., and Chang, G. J. (2009). Humoral immune responses of dengue fever patients using epitopespecific serotype-2 virus-like particle antigens. PLoS ONE 4, e4991. doi: 10.1371/journal.pone.0004991

Dale, C. J., Thomson, S., De Rose, R., Ranasinghe, C., Medveczky, C. J., Pamungkas, J., et al. (2006). Primeboost strategies in DNA vaccines. Methods Mol. Med. 127, 171-197.

Davis, B. S., Chang, G. J., Cropp, B., Roehrig, J. T., Martin, D. A., Mitchell, C. J., etal. (2001). West Nile virus recombinant DNA vaccine protects mouse and horse from virus challenge and expresses in vitro a noninfectious recombinant antigen that can be used in enzyme-linked immunosorbent assays. J. Virol. 75, 4040-4047.

de Alwis, R., Beltramello, M., Messer, W. B., Sukupolvi-Petty, S., Wahala, W. M., Kraus, A., et al. (2011). In-depth analysis of the antibody response of individuals exposed to primary dengue virus infection. PLoS Negl. Trop. Dis. 5, e1188. doi: 10.1371/journal.pntd.0001188

de Alwis, R., Smith, S. A., Olivarez, N. P., Messer, W. B., Huynh, J. P., Wahala, W. M., et al. (2012). Identification of human neutralizing antibodies that bind to complex epitopes on dengue virions. Proc. Natl. Acad. Sci. U.S.A. 109, 7439-7444.

Dejnirattisai, W., Jumnainsong, A., Onsirisakul, N., Fitton, P., Vasanawathana, S., Limpitikul, W., et al. (2010). Cross-reacting antibodies enhance dengue virus infection in humans. Science 328, 745-748.

Ding, H., Tsai, C., Gutierrez, R. A., Zhou, F., Buchy, P., Deubel, V., and Zhou, P. (2011). Superior neutralizing antibody response and protection in mice vaccinated with heterologous DNA prime and virus like particle boost against HPAI H5N1 virus. PLoS ONE 6, e16563. doi: 10.1371/journal.pone. 0016563

Duangchinda, T., Dejnirattisai, W., Vasanawathana, S., Limpitikul, W., Tangthawornchaikul, N., Malasit, P., et al. (2010). Immunodominant Tcell responses to dengue virus NS3 are associated with DHF. Proc. Natl. Acad. Sci. U.S.A. 107, 16922-16927.

Durbin, A. P., and Whitehead, S. S. (2009). Dengue vaccine candidates in development. Curr. Top. Microbiol. Immunol. 338, 129-143.

Durbin, A. P., and Whitehead, S. S. (2011). Next-generation dengue vaccines: novel strategies currently under development. Viruses 3, 1800 1814.
Francis, T. Jr. (1953). Influenza: the new acquayantance. Ann. Intern. Med. 39, 203-221.

Friberg, H., Burns, L., Woda, M., Kalayanarooj, S., Endy, T. P., Stephens, H. A., etal. (2011). Memory CD8+ T cells from naturally acquired primary dengue virus infection are highly cross-reactive. Immunol. Cell Biol. 89, 122-129.

Goncalvez, A. P., Engle, R. E., St Claire, M., Purcell, R. H., and Lai, C. J. (2007). Monoclonal antibodymediated enhancement of dengue virus infection in vitro and in vivo and strategies for prevention. Proc Natl. Acad. Sci. U.S.A. 104, 9422 9427.

Gromowski, G. D., and Barrett, A. D. (2007). Characterization of an antigenic site that contains a dominant type-specific neutralization determinant on the envelope protein domain III (ED3) of dengue 2 virus. Virology 366, 349-360.

Gromowski, G. D., Barrett, N. D., and Barrett, A. D. (2008). Characterization of dengue virus complex-specific neutralizing epitopes on envelope protein domain III of dengue 2 virus. J. Virol. 82, 8828-8837.

Gubler, D. J. (2002). The global emergence/resurgence of arboviral diseases as public health problems. Arch. Med. Res. 33, 330-342.

Gubler, D. J. (2006). Dengue/dengue haemorrhagic fever: history and current status. Novartis Found. Symp. 277, 3-16; discussion 16-22, 71-13, 251-253.

Guenaga, J., Dosenovic, P., Ofek, G. Baker, D., Schief, W. R., Kwong, P. D., et al. (2011). Heterologous epitopescaffold prime:boosting immunofocuses B cell responses to the HIV1 gp41 2F5 neutralization determinant. PLOS ONE 6, e16074. doi: 10.1371/journal.pone.0016074

Guy, B., Barban, V., Mantel, N., Aguirre, M., Gulia, S., Pontvianne, J., et al. (2009). Evaluation of interferences between dengue vaccine serotypes in a monkey model. Am. J. Trop. Med. Hyg. 80, 302-311.

Guy, B., Barrere, B., Malinowski, C., Saville, M., Teyssou, R., and Lang, J. (2011). From research to phase III: preclinical, industrial and clinical development of the Sanofi Pasteur tetravalent dengue vaccine. Vaccine 29, 7229-7241.

Guy, B., Saville, M., and Lang, J. (2010). Development of Sanofi Pasteur tetravalent dengue vaccine. Hum. Vaccin. 6, 1-10.

Guzman, M. G., Halstead, S. B., Artsob, H., Buchy, P., Farrar, J., Gubler, D. J., et al. (2010). Dengue: a continuing global threat. Nat. Rev. Microbiol. 8 , S7-S16.

Halstead, S. B. (1989). Antibody, macrophages, dengue virus infection, shock, and hemorrhage: a pathogenetic cascade. Rev. Infect. Dis. 11(Suppl. 4), S830-S839.

Hawkes, R. A. (1964). Enhancement of the infectivity of arboviruses by specific antisera produced in domestic fowls. Aust. J. Exp. Biol. Med. Sci. 42, 465-482.

Heinz, F. X., and Stiasny, K. (2012). Flaviviruses and flavivirus vaccines. Vaccine 30, 4301-4306.

Henchal, E. A., Mccown, J. M., Burke, D. S., Seguin, M. C., and Brandt, W. E. (1985). Epitopic analysis of antigenic determinants on the surface of dengue- 2 virions using monoclonal antibodies. Am. J. Trop. Med. Hyg. 34 162-169.

Hughes, H. R., Crill, W. D., and Chang, G. J. (2012a). Manipulation of immunodominant dengue virus E protein epitopes reduces potential antibody-dependent enhancement. Virol. J. 9, 115.

Hughes, H. R., Crill, W. D., Davis, B. S., and Chang, G. J. (2012b). A West Nile virus $\mathrm{CD} 4 \mathrm{~T}$ cell epitope improves the immunogenicity of dengue virus serotype 2 vaccines. Virology 424, 129-137.

Kuno, G. (2003). Serodiagnosis of flaviviral infections and vaccinations in humans. Adv. Virus Res. 61, 3-65.

Lai, C. Y., Tsai, W. Y., Lin, S. R. Kao, C. L., Hu, H. P., King, C. C. et al. (2008). Antibodies to envelope glycoprotein of dengue virus during the natural course of infection are predominantly cross-reactive and recognize epitopes containing highly conserved residues at the fusion loop of domain II. J. Virol. 82, 6631-6643.

Laylor, R., Porakishvili, N., De Souza, J. B., Playfair, J. H., Delves, P. J., and Lund, T. (1999). DNA vaccination favours memory rather than effector B cell responses. Clin. Exp. Immunol. $117,106-112$

Libraty, D. H., Endy, T. P., Houng, H. S., Green, S., Kalayanarooj, S. Suntayakorn, S., et al. (2002). Differing influences of virus burden and immune activation on disease severity in secondary dengue- 3 virus infections. J. Infect. Dis. 185, 1213-1221.

Lin, S. R., Zou, G., Hsieh, S. C., Qing, M., Tsai, W. Y., Shi, P. Y., et al. (2011). The helical domains of the stem region of dengue virus envelope protein are involved in both virus assembly and entry. J. Virol. 85, 5159-5171.

Mackenzie, J. S., Gubler, D. J., and Petersen, L. R. (2004). Emerging flaviviruses: the spread and resurgence of Japanese encephalitis, West Nile and dengue viruses. Nat. Med. 10, S98-S109.

Martin, J. E., Pierson, T. C., Hubka, S., Rucker, S., Gordon, I. J., Enama, M. E., et al. (2007). A West Nile virus DNA vaccine induces neutralizing antibody in healthy adults during a phase 1 clinical trial. J. Infect. Dis. 196, 1732-1740.

Mathew, A., and Rothman, A. L. (2008). Understanding the contribution of cellular immunity to dengue disease pathogenesis. Immunol. Rev. 225, 300-313.

Miller, N. (2010). Recent progress in dengue vaccine research and development. Curr. Opin. Mol. Ther. 12, 31-38.

Modis, Y., Ogata, S., Clements, D., and Harrison, S. C. (2003). A ligandbinding pocket in the dengue virus envelope glycoprotein. Proc. Natl. Acad. Sci. U.S.A. 100, 6986-6991.

Morens, D. M. (1994). Antibodydependent enhancement of infection and the pathogenesis of viral disease. Clin. Infect. Dis. 19, 500-512.

Morrison, D., Legg, T. J., Billings, C. W., Forrat, R., Yoksan, S., and Lang, J. (2010). A novel tetravalent dengue vaccine is well tolerated and immunogenic against all 4 serotypes in flavivirus-naive adults. J. Infect. Dis. 201, 370-377.

Murphy, B. R., and Whitehead, S. S. (2011). Immune response to dengue virus and prospects for a vaccine. Annu. Rev. Immunol. 29, 587-619.

Nara, P. L., Tobin, G. J., Chaudhuri, A. R., Trujillo, J. D., Lin, G., Cho, M. W., et al. (2011). How can vaccines against influenza and other viral diseases be made more effective? PLoS Biol. 8, e1000571. doi: 10.1371/journal.pbio. 1000571

Ndifon, W., Wingreen, N. S., and Levin, S. A. (2009). Differential neutralization efficiency of hemagglutinin epitopes, antibody interference, and the design of influenza vaccines. Proc. Natl. Acad. Sci. U.S.A. 106, 87018706.

Petersen, L. R., and Roehrig, J. T. (2007). Flavivirus DNA vaccines-good science, uncertain future. J. Infect. Dis. 196, 1721-1723.

Pierson, T. C., Fremont, D. H., Kuhn, R. J., and Diamond, M. S. (2008). Structural insights into the mechanisms of antibody-mediated neutralization of flavivirus infection: implications for vaccine development. Cell Host Microbe 4, 229-238.

Rodenhuis-Zybert, I. A., Van Der Schaar, H. M., Da Silva Voorham, J. M., Van Der Ende-Metselaar, H., 
Lei, H. Y., Wilschut, J., et al. (2010). Immature dengue virus: a veiled pathogen? PLoS Pathog. 6, el000718. doi: 10.1371/journal.ppat.1000718

Roehrig, J. T., Bolin, R. A., and Kelly, R. G. (1998). Monoclonal antibody mapping of the envelope glycoprotein of the dengue 2 virus, Jamaica. Virology 246, 317-328.

Roehrig, J. T., Risi, P. A., Brubaker, J. R., Hunt, A. R., Beaty, B. J., Trent, D. W., et al. (1994). T-helper cell epitopes on the E-glycoprotein of dengue 2 Jamaica virus. Virology 198, 31-38.

Rothman, A. L. (2004). Dengue: defining protective versus pathologic immunity. J. Clin. Invest. 113, 946-951.

Sabin, A. B. (1952). Research on dengue during WWII. Am. J. Trop. Med. Hyg. 1, 30-50.

Schijns, V. E., Haagmans, B. L., Rijke, E. O., Huang, S., Aguet, M., and Horzinek, M. C. (1994). IFN-gamma receptor-deficient mice generate antiviral Th1-characteristic cytokine profiles but altered antibody responses. J. Immunol. 153, 2029 2037.

Schmitz, J., Roehrig, J., Barrett, A., and Hombach, J. (2011). Next generation dengue vaccines: a review of candidates in preclinical development. Vaccine 29, 7276-7284.

Shresta, S., Sharar, K. L., Prigozhin, D. M., Beatty, P. R., and Harris, E. (2006). Murine model for dengue virus-induced lethal disease with increased vascular permeability. J. Virol. 80, 10208-10217.

Simmons, M., Burgess, T., Lynch, J., and Putnak, R. (2010). Protection against dengue virus by non-replicating and live attenuated vaccines used together in a prime boost vaccination strategy. Virology 396, 280-288.

Sin Leo, Y., Wilder-Smith, A., Archuleta, S., Shek, L., Chong, C. Y., Nam Leong, H., et al. (2012). Immunogenicity and safety of recombinant tetravalent dengue vaccine (CYD-TDV) in individuals aged 2-45 y: Phase II randomized controlled trial in Singapore. Hum. Vaccin. Immunother. 8. [Epub ahead of print].

Stiasny, K., Kiermayr, S., Holzmann, H., and Heinz, F. X. (2006). Cryptic properties of a cluster of dominant flavivirus cross-reactive antigenic sites. J. Virol. 80, 9557-9568.

Sukupolvi-Petty, S., Austin, S. K. Purtha, W. E., Oliphant, T., Nybakken, G. E., Schlesinger, J. J., et al. (2007). Type- and subcomplexspecific neutralizing antibodies against domain III of dengue virus type 2 envelope protein recognize adjacent epitopes. J. Virol. 81 , 12816-12826.

Sun, W., Cunningham, D., Wasserman, S. S., Perry, J., Putnak, J. R., Eckels, K. H., et al. (2009). Phase 2 clinical trial of three formulations of tetravalent live-attenuated dengue vaccine in flavivirus-naive adults. Hum. Vaccin. 5, 33-40.

Thomas, S., Redfern, J. B., Lidbury, B. A., and Mahalingam, S. (2006). Antibody-dependent enhancement and vaccine development. Expert Rev. Vaccines 5, 409-412.

Thomas, S. J. (2011). The necessity and quandaries of dengue vaccine development. J. Infect. Dis. 203, 299-303.

Tobin, G. J., Trujillo, J. D., Bushnell, R. V., Lin, G., Chaudhuri, A. R., Long, J., et al. (2008). Deceptive imprinting and immune refocusing in vaccine design. Vaccine 26, 6189-6199.

Vaughn, D. W., Green, S., Kalayanarooj, S., Innis, B. L., Nimmannitya, S., Suntayakorn, S., et al. (2000). Dengue viremia titer, antibody response pattern, and virus serotype correlate with disease severity. J. Infect. Dis. 181, 2-9.

Wahala, W. M., Huang, C., Butrapet, S., White, L. J., and De Silva, A. M.
(2012). Recombinant dengue type 2 viruses with altered $\mathrm{E}$ protein domain III epitopes are efficiently neutralized by human immune sera. J. Virol. 86 , 4019-4023.

Walker, L. M., and Burton, D. R. (2010). Rational antibody-based HIV-1 vaccine design: current approaches and future directions. Curr. Opin. Immunol. 22, 358-366.

Wei, C. J., Boyington, J. C., Mctamney, P. M., Kong, W. P., Pearce, M. B., Xu, L., et al. (2010). Induction of broadly neutralizing $\mathrm{H} 1 \mathrm{~N}$ influenza antibodies by vaccination. Science 329, 1060-1064.

Welsh, R. M., and Fujinami, R. S. (2007). Pathogenic epitopes, heterologous immunity and vaccine design. Nat. Rev. Microbiol. 5, 555-563.

Welsh, R. M., and Selin, L. K. (2002). No one is naive: the significance of heterologous T-cell immunity. Nat. Rev. Immunol. 2, 417-426.

Welsh, R. M., Selin, L. K., and Szomolanyi-Tsuda, E. (2004). Immunological memory to viral infections. Annu. Rev. Immunol. 22, 711-743.

Williams, K. L., Zompi, S., Beatty, P. R., and Harris, E. (2009). A mouse model for studying dengue virus pathogenesis and immune response. Ann. N. Y. Acad. Sci. 1171(Suppl. 1), E12-E23.

Yauch, L. E., Prestwood, T. R., May, M. M., Morar, M. M., Zellweger, R. M., Peters, B., etal. (2010). CD4+ $\mathrm{T}$ cells are not required for the induction of dengue virus-specific $\mathrm{CD} 8+\mathrm{T}$ cell or antibody responses but contribute to protection after vaccination. J. Immunol. 185 , 5405-5416.

Yauch, L. E., Zellweger, R. M., Kotturi, M. F., Qutubuddin, A., Sidney, J., Peters, B., et al. (2009). A protective role for dengue virus-specific CD8+ T cells. J. Immunol. 182, 4865-4873.
Zellweger, R. M., Prestwood, T. R., and Shresta, S. (2010). Enhanced infection of liver sinusoidal endothelial cells in a mouse model of antibodyinduced severe dengue disease. Cell Host Microbe 7, 128-139.

Zhang, W., Chipman, P. R., Corver, J., Johnson, P. R., Zhang, Y., Mukhopadhyay, S., et al. (2003). Visualization of membrane protein domains by cryoelectron microscopy of dengue virus. Nat. Struct. Biol. 10, 907-912.

Zompi, S., Santich, B. H., Beatty, P. R., and Harris, E. (2012). Protection from secondary dengue virus infection in a mouse model reveals the role of serotype cross-reactive B and T cells. J. Immunol. 188, 404-416.

Conflict of Interest Statement: The findings and conclusions in this article are those of the authors and do not necessarily represent the views of the Centers for Disease Control and Prevention.

Received: 31 August 2012; accepted: 20 October 2012; published online: 08 November 2012.

Citation: Crill WD, Hughes HR, Trainor NB, Davis BS, Whitney MT and Chang G-JJ (2012) Sculpting humoral immunity through dengue vaccination to enhance protective immunity. Front. Immun. 3:334. doi: 10.3389/fimmu.2012.00334

This article was submitted to Frontiers in Microbial Immunology, a specialty of Frontiers in Immunology.

Copyright (c) 2012 Crill, Hughes, Trainor, Davis, Whitney and Chang. This is an open-access article distributed under the terms of the Creative Commons Attribution License, which permits use, distribution and reproduction in other forums, provided the original authors and source are credited and subject to any copyright notices concerning any third-party graphics etc. 